October 31, 2018

\title{
Dyonic Integrable Models
}

\author{
J.F. Gomes, E. P. Gueuvoghlanian, G.M. Sotkov and A.H. Zimerman \\ Instituto de Física Teórica - IFT/UNESP \\ Rua Pamplona 145 \\ 01405-900, São Paulo - SP, Brazil \\ jfg@ift.unesp.br, gueuvogh@ift.unesp.br, sotkov@ift.unesp.br, zimerman@ift.unesp.br
}

\begin{abstract}
A class of non abelian affine Toda models arising from the axial gauged two-loop WZW model is presented. Their zero curvature representation is constructed in terms of a graded Kac-Moody algebra. It is shown that the discrete multivacua structure of the potential together with non abelian nature of the zero grade subalgebra allows soliton solutions with non trivial electric and topological charges. The dressing transformation is employed to explicitly construct one and two soliton solutions and their bound states in terms of the tau functions. A discussion of the classical spectra of such solutions and the time delays are given in detail.
\end{abstract}




\section{Introduction}

The particle-like nonperturbative solutions of the Yang-Mills-Higgs (YMH) model and of the self-dual Yang-Mills (SDYM) equations - monopoles, dyons, instantons, etc, as well as their extended solutions -domain walls (DW) and strings - are known to have many common features with the soliton solutions of certain 1- and 2-D integrable models (IM). Each of these solutions corresponds to specific boundary conditions (b.c.'s) imposed on the 4-D fields, $A_{\mu}^{a}$ and $\phi^{a}$ at space infinities, that in general break translational invariance, Lorentz rotations and the gauge symmetry. As a consequence the 4-D SDYM (or YMH- Bogomolny) equations are reduced to lower dimensional integrable equations. The most representative example is the spherical symmetric 1-monopole of the $S U(r+1)$-YMH model described in terms of specific solutions of $1-\mathrm{D} S U(r+1)$ - abelian Toda theory [1].

Recently, solitons of 2-D $N=2$ supersymmetric $C P^{r}$ model ( and its mirror dual $N=2$ $S U(r+1)$ abelian affine Toda model )have been identified as BPS domain walls of 4-D $N=1$ supersymmetric $S U(r+1)$ YM theory [2], [3], [4]. Since large $r$ limits of $N=1$ SUSY YM and of the ordinary QCD are believed to coincide [5], one expects that domain walls of non SUSY YMH (for large $r$ and maximal breaking $\left.S U(r+1) \rightarrow U(1)^{r}\right)$ to be related to solitons of 2-D $S U(r+1)$ abelian affine Toda model. The known exact solitonic results of the 2-D IMs can than be used to describe nonperturbative properties of the domain walls [2], [3]. The domain wall's tensions, for instance are proportional to the soliton masses. DW's junctions are related to the multisolitons, breathers ans excited solitons, etc.

In (super)string aproach to 4-D YMH theory, the DW's appear as specific D2-branes where QCD string can end [4]. An interesting "soliton solution" of 10-D (or 26-D) low energy effective field theory requires together with simple DW's, for instance, $U(1)$ charged DW's, $n$ - coincident D2-branes, nonstatic (but stationary ) DW's, etc. The explicit description of such DW's in terms of 2-D solitons addresses the problem of constructing new families of IMs of dyonic type admiting $U(1)$ charged topological solitons, global (or local ) $U(1)$ Noether symmetry, CPT breaking $\theta$-terms (see for instance [6]) The abelian affine Toda theories are examples of IM's satisfying none of these requirements. It is natural to seek for such dyonic IM's within the class of non-abelian affine Toda models [10, 13. Integrable models with $U(1)$ (or more general $U(1)^{r}$ ) symmetry such as the Lund-Regge (LR) (or complex SineGordon, CSG) model [7] and its homogeneous space SG generalizations [8] appear to be of purely electric type: their solitons are $U(1)^{r}$ charged but nontopological. The simplest IM of dyonic type with Lagrangean

$$
\begin{aligned}
\mathcal{L} & =\frac{1}{2} \sum_{i, j=2}^{r} K_{i j} \partial^{\mu} \varphi_{i} \partial_{\mu} \varphi_{j}+e^{-\beta \varphi_{2}} \frac{\left(\partial^{\mu} \psi \partial_{\mu} \chi+\epsilon^{\mu \nu} \partial_{\mu} \psi \partial_{\nu} \chi\right)}{1+\beta^{2} \frac{n+1}{2 n} \psi \chi e^{-\beta \varphi_{2}}} \\
& -\frac{\mu^{2}}{\beta^{2}}\left(\sum_{i=2}^{r} e^{-\beta K_{i j} \varphi_{j}}+e^{\beta\left(\varphi_{r}+\varphi_{2}\right)}\left(1+\beta^{2} \psi \chi e^{-\beta \varphi_{2}}\right)-r\right)
\end{aligned}
$$

$K_{i j}=2 \delta_{i j}-\delta_{i, j-1}-\delta_{i, j+1}, \quad i, j=2, \cdots r$, have been studied in our recent paper [9]. For imaginary coupling $\beta \rightarrow i \beta_{0}$ its potential has $r-1$ distinct vacua, $Z_{2} \otimes Z_{r-1}$ symmetry and as a consequence the model (1.1) admits, together with the neutral topological solitons (of the $A_{r-1}^{(1)}$ abelian affine Toda type ), the $U(1)$ charged topological 1-solitons with spectra quite 
similar to the YMH dyons [9]. These dyonic type of solitons share many of the properties of the charged DWs in the $N=1$ SYM for nonmaximal breaking $S U(r+1) \rightarrow S U(2) \otimes U(1)^{r-1}$.

The present paper is devoted to the systematic construction of IM of dyonic type and of their multisoliton and breather solutions. The method used in the derivation of their action represents a functional integral version of the Leznov-Saveliev algebraic approach [10] to the $\hat{\mathcal{G}}_{r}$ non-abelian (NA) affine Toda IM. It is explained in Sect. 2 that a large class of 2-D IM can be defined as axial (or vector) gauged two loop WZW models $\hat{H}_{-} \backslash \hat{G}_{r} / \hat{H}_{+}$ 111 by extending the methods for constructing conformal $\sigma$-models and conformal NA-Toda 16] to include all integrable (non conformal ) perturbations. As it is well known each affine (Kac-Moody) algebra $\hat{G}_{r}$ gives rise to many different IM's depending on the choice of specific graded structure defined in terms of a grading operator denoted by $Q$ and by the constant grade \pm 1 elements $\epsilon_{ \pm}$(or $\pm p$, for higher grading model).

The zero grade (factor) group $\Gamma_{0}=\hat{H}_{-} \backslash \hat{G}_{r} / \hat{H}_{+}$is parametrized by the (physical ) fields (say $\left.\varphi_{i}, \psi, \chi\right)$ present in the action $\mathbb{\Psi}$. The elements of the zero grade subalgebras $\mathcal{G}_{0}^{0} \subset \mathcal{G}_{0}$ such that $\left[\mathcal{G}_{0}^{0}, \epsilon_{ \pm}\right]=0$, generates the (Noether) symmetries of the corresponding IM. They are by construction, local symmetries and when gauged away, give rise to the so called singular NA affine Toda with $\mathcal{G}_{0}^{0}$ as the global symmetry group. The soliton charges are shown to depend upon $\mathcal{G}_{0}^{0}$ and upon the specific form of $\epsilon_{ \pm}$which defines the potential of our IM $V=\operatorname{Tr}\left(B^{-1} \epsilon_{+} B \epsilon_{-}\right), \quad\left(B \in G_{0} / G_{0}^{0}\right)$ and on the boundary condition (b.c.'s $)$ imposed upon the fields. The model of magnetic type has no Noether charges $\left\{\mathcal{G}_{0}^{0}\right\}=\emptyset$ and $\epsilon_{ \pm}$are chosen in such way that provides nontrivial zeros of the potential $V$. They have maximal number of magnetic (i.e. topological) charges $m_{i}: \Lambda=\frac{2 \pi}{\beta} \sum_{i=1}^{r} m_{i} \alpha_{i}^{v}$. The electric type IM are characterized by the maximal number of Noether charges $\mathcal{G}_{0}^{0} \equiv U(1)^{r}$ that restrict $\epsilon_{ \pm}=\sum_{i=1}^{r} \mu_{i} h_{i}^{( \pm 1)},(Q=\hat{d})$, (homogeneous gradation) and for the standard b.c. (all fields vanish at $\pm \infty$ ) leads to potentials with trivial magnetic charges. The main characteristic of the IM of dyonic type is that only a part of the Cartan subalgebra $\mathcal{H}_{r}=\oplus_{1}^{r} U(1)$, (i.e. $\left.\mathcal{G}_{0}^{0}=U(1)^{s}, 0 \leq s \leq r\right)$ generates Noether symmetries (i.e. the number of $U(1)$ charges is $0 \leq s \leq r)$ and the rest $r-s$ can be converted into magnetic charges $m_{a}, a=s+1, \cdots, r$ if $\epsilon_{ \pm}$are appropriately choosen. The generic type $U(1)$ dyonic IM (i.e. $s=1$ ) is defined by

$$
Q=\tilde{h} \hat{d}+\sum_{i \neq a}^{r} \frac{2 \lambda_{i} \cdot H^{(0)}}{\alpha_{i}^{2}}, \quad \epsilon_{ \pm}=\sum_{i \neq a} \mu_{i} E_{ \pm \alpha_{i}}^{(0)}+\sum_{l} \mu_{0 l} E_{\mp \beta_{0 l}}^{( \pm 1)}
$$

$\mathcal{G}_{0}^{0}=\left\{\lambda_{a} \cdot H^{(0)}, 1 \leq a \leq r,\right\}$ a fixed, $\alpha_{i}$ are the simple roots of $\hat{G}_{r}$ and $\beta_{0 l}$ are specific composite roots. The exact algebraic criterium for the construction of $U(1)$ dyonic IMs of axial type is given in Sect. 2. The condition for absence (or presence ) of CPT-breaking terms as well as the derivation of the CPT-invariant dyonic IMs of the vector type ( the Tduals of (1.1)) are presented in [9] and [25]. The IM (1.1) corresponds to $\hat{G}_{r}=A_{r}^{(1)}, a=1$ (i.e. $\left.\mathcal{G}_{0}^{0}=\lambda_{1} \cdot H^{(0)}\right), \beta_{0}=\alpha_{2}+\alpha_{3}+\cdots+\alpha_{r}, \tilde{h}=r$ and $\mu_{i}=\mu_{0 l}=\mu$.

We further choose to study the IM (1.1) as representative of the family of a single $U(1)$ charge IM described in Sect. 2. The remaining part of the paper (Sects. 3-8) contains the explicit constructions of all 1- and 2- soliton solutions and their bound states by employing

\footnotetext{
${ }^{1}$ Note that the nonconformal affine NA Toda models are constructed in terms of the centerless Kac-Moody algebra, i.e. $\hat{c}=0$
} 
the method of dressing transformation (or "vertex operators", or "soliton specialization" " $\tau$-function"). The complete list of "irreducible" particle-like solutions contains together with the $U(1)$ charged topological 1-soliton also neutral topological 1-solitons of species $a$ [20], and their bound states:

- "2-neutral vertex" solution, that coincides with $A_{r-1}^{(1)}$ abelian affine Toda bound states [12].

- "3-vertex" describing the one charged 1-soliton and one neutral 1-soliton bound state

- "4-vertex" representing a bound state of two charged 1-(anti) solitons.

An important feature of the neutral and charged (multi) solitons and bound states of IMs (1.1) is the so called vacuum degeneration. It consists in the existence of soliton solutions with same energy, momentun, mass, electric charge, but with different number (moduli ) parameters and different form of the solutions (i.e. $\tau$-functions ). They are constructed by appropriated modification of the defining constant element $g$, characteristic of the dressing method (see Sect. 4.3 and 5).

The scattering phase shifts (time delays ) are derived in Sect.6 from the explicit form of the various 2 -soliton - antisoliton solutions.

\section{Construction of the Model}

The generic NA Toda models are classified according to a $\mathcal{G}_{0} \subset \mathcal{G}$ embedding induced by the grading operator $Q$ decomposing an finite or infinite Lie algebra $\mathcal{G}=\oplus_{i} \mathcal{G}_{i}$ where $\left[Q, \mathcal{G}_{i}\right]=i \mathcal{G}_{i}$ and $\left[\mathcal{G}_{i}, \mathcal{G}_{j}\right] \subset \mathcal{G}_{i+j}$. A group element $g$ can then be written in terms of the Gauss decomposition as

$$
g=N B M
$$

where $N=\exp \mathcal{G}_{<}, B=\exp \mathcal{G}_{0}$ and $M=\exp \mathcal{G}_{>}$. The physical fields lie in the zero grade subgroup $B$ and the models we seek correspond to the coset $H_{-} \backslash G / H_{+}$, for $H_{ \pm}$generated by positive/negative grade operators.

For consistency with the hamiltonian reduction formalism, the phase space of the Ginvariant WZNW model is reduced by specifying the constant generators $\epsilon_{ \pm}$of grade \pm 1 . In order to derive an action for $B$, invariant under

$$
g \longrightarrow g^{\prime}=\alpha_{-} g \alpha_{+},
$$

where $\alpha_{ \pm}(z, \bar{z})$ lie in the positive/negative grade subgroup we have to introduce a set of auxiliary gauge fields $A \in \mathcal{G}_{<}$and $\bar{A} \in \mathcal{G}_{>}$transforming as

$$
A \longrightarrow A^{\prime}=\alpha_{-} A \alpha_{-}^{-1}+\alpha_{-} \partial \alpha_{-}^{-1}, \quad \bar{A} \longrightarrow \bar{A}^{\prime}=\alpha_{+}^{-1} \bar{A} \alpha_{+}+\bar{\partial} \alpha_{+}^{-1} \alpha_{+},
$$

where $z=\frac{1}{2}(t+x), \bar{z}=\frac{1}{2}(t-x)$. The resulting action is the $G / H\left(=H_{-} \backslash G / H_{+}\right)$gauged WZNW

$$
\begin{aligned}
S_{G / H}(g, A, \bar{A}) & =S_{W Z N W}(g) \\
& -\frac{k}{2 \pi} \int d^{2} x \operatorname{Tr}\left(A\left(\bar{\partial} g g^{-1}-\epsilon_{+}\right)+\bar{A}\left(g^{-1} \partial g-\epsilon_{-}\right)+A g \bar{A} g^{-1}\right) .
\end{aligned}
$$


Since the action $S_{G / H}$ is $H$-invariant, we may choose $\alpha_{-}=N^{-1}$ and $\alpha_{+}=M^{-1}$. From the orthogonality of the graded subpaces, i.e. $\operatorname{Tr}\left(\mathcal{G}_{i} \mathcal{G}_{j}\right)=0, i+j \neq 0$, we find

$$
\begin{aligned}
S_{G / H}(g, A, \bar{A}) & =S_{G / H}\left(B, A^{\prime}, \bar{A}^{\prime}\right) \\
& =S_{W Z N W}(B)-\frac{k}{2 \pi} \int d^{2} x \operatorname{Tr}\left[-A^{\prime} \epsilon_{+}-\bar{A}^{\prime} \epsilon_{-}+A^{\prime} B \bar{A}^{\prime} B^{-1}\right],
\end{aligned}
$$

where

$$
S_{W Z N W}=-\frac{k}{4 \pi} \int d^{2} x \operatorname{Tr}\left(g^{-1} \partial g g^{-1} \bar{\partial} g\right)+\frac{k}{24 \pi} \int_{D} \epsilon^{i j k} \operatorname{Tr}\left(g^{-1} \partial_{i} g g^{-1} \partial_{j} g g^{-1} \partial_{k} g\right) d^{3} x,
$$

and the topological term denotes a surface integral over a ball $D$ identified as space-time.

Action (2.4) describes the non singular Toda models among which we find the Conformal and the Affine abelian Toda models where $Q=\sum_{i=1}^{r} \frac{2 \lambda_{i} \cdot H}{\alpha_{i}^{2}}, \quad \epsilon_{ \pm}=\sum_{i=1}^{r} \mu_{ \pm i} E_{ \pm \alpha_{i}}$ and $Q=$ $h d+\sum_{i=1}^{r} \frac{2 \lambda_{i} \cdot H^{(0)}}{\alpha_{i}^{2}}, \quad \epsilon_{ \pm}=\sum_{i=1}^{r} \mu_{ \pm i} E_{ \pm \alpha_{i}}^{(0)}+\mu_{0} E_{\mp \psi}^{( \pm 1)}$ respectively, $\psi$ denote the highest root, $\lambda_{i}$ are the fundamental weights and $h$ the coxeter number of $\mathcal{G}$.

Performing the integration over the auxiliary fields $A$ and $\bar{A}$, the functional integral

$$
Z_{ \pm}=\int D A D \bar{A} \exp \left(F_{ \pm}\right)
$$

where

$$
F_{ \pm}=-\frac{k}{2 \pi} \int\left(\operatorname{Tr}\left(A-B \epsilon_{-} B^{-1}\right) B\left(\bar{A}-B^{-1} \epsilon_{+} B\right) B^{-1}\right) d^{2} x
$$

yields the effective action

$$
S=S_{W Z N W}(B)+\frac{k}{2 \pi} \int \operatorname{Tr}\left(\epsilon_{+} B \epsilon_{-} B^{-1}\right) d^{2} x
$$

The action (2.8) describes integrable perturbations of the $\mathcal{G}_{0}$-WZNW model. Those perturbations are classified in terms of the possible constant grade \pm 1 operators $\epsilon_{ \pm}$.

More interesting cases arises in connection with non abelian embeddings $\mathcal{G}_{0} \subset \mathcal{G}$. In particular, if we supress one of the fundamental weights from $Q$, the zero grade subspace $\mathcal{G}_{0}$, acquires a nonabelian structure $\operatorname{sl}(2) \otimes u(1)^{r a n k \mathcal{G}-1}$. Let us consider for instance $Q=$ $h^{\prime} d+\sum_{i \neq a}^{r} \frac{2 \lambda_{i} \cdot H}{\alpha_{i}^{2}}$, where $h^{\prime}=0$ or $h^{\prime} \neq 0$ 己 corresponding to the Conformal or Affine nonabelian (NA) Toda respectively. The absence of $\lambda_{a}$ in $Q$ prevents the contribution of the simple root step operator $E_{\alpha_{a}}^{(0)}$ in constructing $\epsilon_{+}$. It in fact, allows for reducing the phase space even further. This fact can be understood by enforcing the nonlocal constraint $J_{Y \cdot H}=\bar{J}_{Y \cdot H}=0$ where $Y$ is such that $\left[Y \cdot H, \epsilon_{ \pm}\right]=0$ and $J=g^{-1} \partial g$ and $\bar{J}=-\bar{\partial} g g^{-1}$. Those generators of $\mathcal{G}_{0}$ commuting with $\epsilon_{ \pm}$define a subalgebra $\mathcal{G}_{0}^{0} \subset \mathcal{G}_{0}$. Such subsidiary constraint is incorporated into the action by requiring symmetry under [16]

$$
g \longrightarrow g^{\prime}=\alpha_{0} g \alpha_{0}^{\prime}
$$

where we shall consider $\alpha_{0}^{\prime}=\alpha_{0}(z, \bar{z}) \in \mathcal{G}_{0}^{0}$, i.e., axial symmetry (the vector gauging is obtained by choosing $\alpha_{0}^{\prime}=\alpha_{0}{ }^{-1}(z, \bar{z}) \in \mathcal{G}_{0}^{0}$, see for instance the second ref. in [25]). Auxiliary

\footnotetext{
${ }^{2}$ For the Kac-Moody case we are suppressing the index (0) in defining the Cartan subalgebra of $\mathcal{G}$
} 
gauge fields $A_{0}=a_{0} Y \cdot H$ and $\bar{A}_{0}=\bar{a}_{0} Y \cdot H \in \mathcal{G}_{0}^{0}$ are introduced to construct an invariant action under transformations (2.9)

$$
\begin{aligned}
S\left(B, A_{0}, \bar{A}_{0}\right) & =S\left(g_{0}^{f}, A^{\prime}{ }_{0}, \bar{A}^{\prime}{ }_{0}\right)=S_{W Z N W}(B)+\frac{k}{2 \pi} \int \operatorname{Tr}\left(\epsilon_{+} B \epsilon_{-} B^{-1}\right) d^{2} x \\
& -\frac{k}{2 \pi} \int \operatorname{Tr}\left(A_{0} \bar{\partial} B B^{-1}+\bar{A}_{0} B^{-1} \partial B+A_{0} B \bar{A}_{0} B^{-1}+A_{0} \bar{A}_{0}\right) d^{2} x
\end{aligned}
$$

where the auxiliary fields transform as

$$
A_{0} \longrightarrow A_{0}^{\prime}=A_{0}-\alpha_{0}^{-1} \partial \alpha_{0}, \quad \bar{A}_{0} \longrightarrow \bar{A}_{0}^{\prime}=\bar{A}_{0}-\bar{\partial} \alpha_{0}^{\prime}\left(\alpha_{0}^{\prime}\right)^{-1}
$$

Such residual gauge symmetry allows us to eliminate an extra field associated to $Y \cdot H$. Notice that the physical fields $g_{0}^{f}$ lie in the coset $\mathcal{G}_{0} / \mathcal{G}_{0}^{0}=\left(s l(2) \otimes u(1)^{r a n k \mathcal{G}-1}\right) / u(1)$ of dimension $\operatorname{rank\mathcal {G}}+1$ and are classified according to the gradation $Q$. It therefore follows that $S\left(B, A_{0}, \bar{A}_{0}\right)=S\left(g_{0}^{f}, A_{0}^{\prime}, \bar{A}_{0}^{\prime}\right)$.

In [15] a detailed study of the gauged WZNW construction for finite dimensional Lie algebras leading to Conformal NA Toda models was presented. The study of its symmetries was given in refs. [16. Here we generalize the construction of ref. [15] to infinite dimensional Lie algebras leading to NA Affine Toda models characterized by the broken conformal symmetry and by the presence of solitons.

Consider the Kac-Moody algebra $\widehat{\mathcal{G}}$

$$
\begin{aligned}
& {\left[T_{m}^{a}, T_{n}^{b}\right]=f^{a b c} T_{m+n}^{c}+\hat{c} m \delta_{m+n} \delta^{a b}} \\
& {\left[\hat{d}, T_{n}^{a}\right]=n T_{n}^{a} ; \quad\left[\hat{c}, T_{n}^{a}\right]=[\hat{c}, \hat{d}]=0}
\end{aligned}
$$

The NA Toda models we shall be constructing are associated to gradations of the type $Q_{a}\left(h^{\prime}\right)=h_{a}^{\prime} d+\sum_{i \neq a}^{r} \frac{2 \lambda_{i} \cdot H^{(0)}}{\alpha_{i}^{2}}$, where $h_{a}^{\prime}$ is chosen such that the gradation, $Q_{a}\left(h^{\prime}\right)$, acting on infinite dimensional Lie algebra $\hat{\mathcal{G}}$ ensures that the zero grade subgroup $\mathcal{G}_{0}$ coincides with its counterpart obtained with $Q_{a}\left(h^{\prime}=0\right)$ acting on the Lie algebra $\mathcal{G}$ of finite dimension apart from two commuting generators $\hat{c}$ and $\hat{d}$. Since they commute with $\mathcal{G}_{0}$, the kinetic part decouples such that the conformal and the affine singular NA-Toda models differ only by the potential term characterized by $\hat{\epsilon}_{ \pm}$.

The integration over the auxiliary gauge fields $A$ and $\bar{A}$ requires explicit parametrization of $B$.

$$
B=\exp \left(\tilde{\chi} E_{-\alpha_{a}}^{(0)}\right) \exp \left(R \sum_{i=1}^{r} Y_{i} H_{i}^{(0)}+\Phi(H)+\nu \hat{c}+\eta \hat{d}\right) \exp \left(\tilde{\psi} E_{\alpha_{a}}^{(0)}\right)
$$

where $\Phi(H)=\sum_{j=1}^{r} \sum_{i=2}^{r} \varphi_{i} X_{i}^{j} H_{j}{ }^{(0)}$, where $\sum_{j=1}^{r} Y_{j} X_{i}^{j}=0, i=2, \cdots, r$. After gauging away the nonlocal field $R$, the factor group element becomes

$$
g_{0}^{f}=\exp \left(\chi E_{-\alpha_{a}}^{(0)}\right) \exp (\Phi(H)+\nu \hat{c}+\eta \hat{d}) \exp \left(\psi E_{\alpha_{a}}^{(0)}\right)
$$


where $\chi=\tilde{\chi} e^{\frac{1}{2}\left(Y \cdot \alpha_{a}\right) R}, \quad \psi=\tilde{\psi} e^{\frac{1}{2}\left(Y \cdot \alpha_{a}\right) R}$. We therefore get for the zero grade component

$$
\begin{aligned}
F_{0} & =-\frac{k}{2 \pi} \int \operatorname{Tr}\left(A_{0} \bar{\partial} g_{0}^{f}\left(g_{0}^{f}\right)^{-1}+\bar{A}_{0}\left(g_{0}^{f}\right)^{-1} \partial g_{0}^{f}+A_{0} g_{0}^{f} \bar{A}_{0}\left(g_{0}^{f}\right)^{-1}+A_{0} \bar{A}_{0}\right) d^{2} x \\
& =-\frac{k}{2 \pi} \int\left(a_{0} \bar{a}_{0} 2 Y^{2} \Delta-2\left(\frac{\alpha_{a} \cdot Y}{\alpha_{a}^{2}}\right)\left(\bar{a}_{0} \psi \partial \chi+a_{0} \chi \bar{\partial} \psi\right) e^{\Phi\left(\alpha_{a}\right)}\right) d^{2} x
\end{aligned}
$$

where $\Delta=1+\frac{\left(Y \cdot \alpha_{a}\right)^{2}}{2 Y^{2}} \psi \chi e^{\Phi\left(\alpha_{a}\right)},\left[\Phi(H), E_{\alpha_{a}}^{(0)}\right]=\Phi\left(\alpha_{a}\right) E_{\alpha_{a}}^{(0)}$.

The effective action is obtained by integrating over the auxiliary fields $A_{0}, \bar{A}_{0}$,

$$
Z_{0}=\int D A_{0} D \bar{A}_{0} \exp \left(F_{0}\right)
$$

The total action (2.10) is therefore given as

$$
S=-\frac{k}{4 \pi} \int\left(\operatorname{Tr}(\partial \Phi(H) \bar{\partial} \Phi(H))+\frac{2 \bar{\partial} \psi \partial \chi}{\Delta} e^{\Phi\left(\alpha_{a}\right)}+\partial \eta \bar{\partial} \nu+\partial \nu \bar{\partial} \eta-2 \operatorname{Tr}\left(\hat{\epsilon_{+}} g_{0}^{f} \hat{\epsilon_{-}}\left(g_{0}^{f}\right)^{-1}\right)\right) d^{2} x
$$

Note that the second term in (2.16) contains both symmetric and antisymmetric parts:

$$
\frac{e^{\Phi\left(\alpha_{a}\right)}}{\Delta} \bar{\partial} \psi \partial \chi=\frac{e^{\Phi\left(\alpha_{a}\right)}}{\Delta}\left(g^{\mu \nu} \partial_{\mu} \psi \partial_{\nu} \chi+\epsilon^{\mu \nu} \partial_{\mu} \psi \partial_{\nu} \chi\right)
$$

where $g_{\mu \nu}$ is the 2-D metric of signature $g_{\mu \nu}=\operatorname{diag}(1,-1), \epsilon^{\mu \nu}$ is the totally antisymmetric tensor $\epsilon^{01}=1$. For $n=1\left(\mathcal{G} \equiv A_{1}, \Phi\left(\alpha_{1}\right)\right.$ is zero $)$ the antisymmetric term is a total derivative:

$$
\epsilon^{\mu \nu} \frac{\partial_{\mu} \psi \partial_{\nu} \chi}{1+\psi \chi}=\frac{1}{2} \epsilon^{\mu \nu} \partial_{\mu}\left(\ln \{1+\psi \chi\} \partial_{\nu} \ln \frac{\chi}{\psi}\right)
$$

and it can be neglected. This $A_{1}$-NA-Toda model (in the conformal case), is known to describe the 2-D black hole solution for (2-D) string theory 18. The $\mathcal{G}$-NA conformal Toda model can be used in the description of specific $(n+1)$-dimensional black string theories [19], with (n-1)-flat and 2-non flat directions $\left(g^{\mu \nu} G_{a b}(X) \partial_{\mu} X^{a} \partial_{\nu} X^{b}, X^{a}=\left(\psi, \chi, \varphi_{i}\right)\right)$, containing axions $\left(\epsilon^{\mu \nu} B_{a b}(X) \partial_{\mu} X^{a} \partial_{\nu} X^{b}\right)$ and tachions $\left(\exp \left\{-k_{i j} \varphi_{j}\right\}\right)$, as well. The affine $A_{1}$-NA Toda theory with $\epsilon_{ \pm}=H^{( \pm)}$corresponds to the Lund-Regge model describing charged solitons [24.

It is clear that the presence of the $e^{\Phi\left(\alpha_{a}\right)}$ in $(2.16)$ is responsible for the antisymmetric tensor generating the axionic terms. On the other hand, notice that $\Phi\left(\alpha_{a}\right)$ depends upon the subsidiary nonlocal constraint $J_{Y \cdot H}=\bar{J}_{Y \cdot H}=0$ and hence upon the choice of the vector $Y$. It is defined to be orthogonal to all roots contained in $\epsilon_{ \pm}$. A Lie algebraic condition for the absence of axionic terms was found in [15] and has provided a construction of a family of torsionless NA Toda models in [25].

The action (2.16) is invariant under the global $U(1)$ transformation

$$
\psi \rightarrow e^{i \epsilon} \psi, \quad \chi \rightarrow e^{-i \epsilon} \chi
$$


The corresponding Noether current is

$$
J^{\mu}=-\frac{i k}{2 \pi} \frac{e^{\Phi\left(\alpha_{a}\right)}}{\Delta}\left\{\psi\left(g^{\nu \mu} \partial_{\nu} \chi-\epsilon^{\nu \mu} \partial_{\nu} \chi\right)-\chi\left(g^{\nu \mu} \partial_{\nu} \psi+\epsilon^{\nu \mu} \partial_{\nu} \psi\right)\right\}
$$

and the electric charge is given in terms of the nonlocal field $R$ defined below in (3.2) as

$$
Q=\int J_{o} d x=-\frac{i k}{\pi}\left(\frac{r}{r+1}\right)[R(\infty)-R(-\infty)]
$$

Apart from the global $U(1)$ symmetry (2.19) there is a discrete set of field transformations leaving the action (2.16) unchanged. Such transformations (for imaginary $\beta, \beta \rightarrow i \beta_{0}$ ) give rise to multivacua configuration and hence to nontrivial topological charges

$$
\begin{aligned}
Q_{j} & =\int_{-\infty}^{+\infty} J_{j}^{0} d x, \quad J_{j}^{\mu}=\frac{1}{2 \pi i} \epsilon^{\mu \nu} \partial_{\nu} \varphi_{j}, \quad j=2, \cdots r \\
Q_{\theta} & =\int_{-\infty}^{+\infty} J_{\theta}^{0} d x \quad J_{\theta}^{\mu}=-i \frac{1}{2 \beta^{2}} \epsilon^{\mu \nu} \partial_{\nu} \ln \left(\frac{\chi}{\psi}\right)
\end{aligned}
$$

Let us explicitly consider the $A_{r}^{(1)}$ model described by the Lagrangean density (3.10) (with fields rescaled by $\varphi_{i} \rightarrow \beta \varphi_{i}, \chi \rightarrow \beta \chi, \psi \rightarrow \beta \psi, \beta^{2}=-\frac{2 \pi}{k}$ ) invariant under the following set of discrete transformations,

$$
\varphi_{j}^{\prime}=\varphi_{j}+\frac{2 \pi(j-1) N}{\beta_{0} r}, j=2, \cdots r, \quad \chi^{\prime}=e^{i \pi\left(\frac{N}{r}+s_{2}\right)} \chi, \quad \psi^{\prime}=e^{i \pi\left(\frac{N}{r}+s_{1}\right)} \psi
$$

where $s_{1}, s_{2}$ are both even or odd integers and the following CP transformations $(\mathrm{P}: x \rightarrow-x)$

$$
\varphi_{j}^{\prime \prime}=\varphi_{j}, j=2, \cdots r, \quad \chi^{\prime \prime}=\psi, \quad \psi^{\prime \prime}=\chi
$$

The minimum of the potential ( for the choice $\eta=0$ ) corresponds to the following field configuration

$$
\varphi_{j}^{(N)}=\frac{2 \pi(j-1) N}{\beta_{0} r}, \quad \theta^{(L)}=\frac{1}{2 i \beta_{0}} \ln \left(\frac{\chi}{\psi}\right)=\frac{\pi L}{\beta_{0}}, \quad \rho^{(0)}=0 \quad j=2, \cdots r
$$

where $N, L$ are arbitrary integers, and the new fields $\theta$ and $\rho$ are defined as

$$
\psi=\frac{1}{\beta_{0}} e^{i \beta_{0}\left(\frac{1}{2} \varphi_{2}-\theta\right)} \sinh \left(\beta_{0} \rho\right), \quad \chi=\frac{1}{\beta_{0}} e^{i \beta_{0}\left(\frac{1}{2} \varphi_{2}+\theta\right)} \sinh \left(\beta_{0} \rho\right)
$$

In fact eqns, (2.25) also represent constant solutions of the eqns. of motion (3.6)-(3.9) which allows us to derive the values of the topological charge (2.22):

$$
Q_{j}=\frac{1}{r}(j-1)\left(N_{+}-N_{-}\right), \quad Q_{\theta}=-\frac{\pi}{\beta_{0}^{2}}\left(L_{+}-L_{-}\right)
$$




\section{Zero Curvature and Equations of Motion}

The equations of motion for the NA Toda models are known to be of the form [10]

$$
\bar{\partial}\left(B^{-1} \partial B\right)+\left[\hat{\epsilon_{-}}, B^{-1} \hat{\epsilon_{+}} B\right]=0, \quad \partial\left(\bar{\partial} B B^{-1}\right)-\left[\hat{\epsilon_{+}}, B \hat{\epsilon_{-}} B^{-1}\right]=0
$$

The subsidiary constraint $J_{Y \cdot H^{(0)}}=\operatorname{Tr}\left(B^{-1} \partial B Y \cdot H^{(0)}\right)$ and $\bar{J}_{Y \cdot H^{(0)}}=\operatorname{Tr}\left(\bar{\partial} B B^{-1} Y \cdot H^{(0)}\right)=0$ can be consistently imposed since $\left[Y \cdot H^{(0)}, \hat{\epsilon_{ \pm}}\right]=0$ as can be obtained from (3.1) by taking the trace with $Y . H^{(0)}$. Solving those equations for the nonlocal field $R$ yields,

$$
\partial R=\left(\frac{Y \cdot \alpha_{a}}{Y^{2}}\right) \frac{\psi \partial \chi}{\Delta} e^{\Phi\left(\alpha_{a}\right)}, \quad \bar{\partial} R=\left(\frac{Y \cdot \alpha_{a}}{Y^{2}}\right) \frac{\chi \bar{\partial} \psi}{\Delta} e^{\Phi\left(\alpha_{a}\right)}
$$

The equations of motion for the fields $\psi, \chi$ and $\varphi_{i}, i=2, \cdots, r$ obtained from (3.1) after imposing the subsidiary constraints (3.2) coincide precisely with the Euler-Lagrange equations derived from (2.16). Alternatively, (3.1) admits a zero curvature representation $\partial \bar{A}-\bar{\partial} A+[A, \bar{A}]=0$ where

$$
A=B \hat{\epsilon_{-}} B^{-1}, \quad \bar{A}=-\hat{\epsilon_{+}}-\bar{\partial} B B^{-1}
$$

Whenever the constraints (3.2) are incorporated into $A$ and $\bar{A}$ in (3.3), equations (3.1) yields the zero curvature representation of the NA singular Toda models.

We shall be considering $\hat{\mathcal{G}}=A_{r}^{(1)}, Q=r \hat{d}+\sum_{i=2}^{r} 2 \frac{\lambda_{i} \cdot H^{(0)}}{\alpha_{i}^{2}}, \sum_{i=1}^{r} Y_{i} H_{i}^{(0)}=2 \frac{\lambda_{1} \cdot H^{(0)}}{\alpha_{1}^{2}}$, $\sum_{j=1}^{r} X_{i}^{j} H_{j}^{(0)}=h_{i}^{(0)}=\frac{2 \alpha_{i} \cdot H^{(0)}}{\alpha_{i}^{2}}$ and $\hat{\epsilon_{ \pm}}=\mu\left(\sum_{i=2}^{r} E_{ \pm \alpha_{i}}^{(0)}+E_{\mp\left(\alpha_{2}+\cdots+\alpha_{r}\right)}^{( \pm 1)}\right), \mu>0$.

Using the explicit parametrization of $B$ given in (2.12), we find, in a systematic manner, the following form for $A$ and $\bar{A}$

$$
\begin{aligned}
A & =\mu\left(\sum_{i=2}^{r} e^{-\sum_{j=2}^{r} K_{i, j} \varphi_{j}} E_{-\alpha_{i}}^{(0)}-\chi e^{-\frac{1}{2} R-2 \varphi_{2}+\varphi_{3}} E_{-\alpha_{1}-\alpha_{2}}^{(0)}\right. \\
& \left.+\psi e^{\frac{1}{2} R+\varphi_{r}-\eta} E_{\alpha_{1}+\cdots \alpha_{r}}^{(-1)}+\left(1+\psi \chi e^{-\varphi_{2}}\right) E_{\alpha_{2}+\cdots+\alpha_{r}}^{(-1)} e^{\varphi_{2}+\varphi_{r}-\eta}\right)
\end{aligned}
$$

and

$$
\begin{aligned}
\bar{A} & =\mu\left(-\sum_{i=2}^{r} E_{\alpha_{i}}^{(0)}-E_{-\alpha_{2}-\ldots-\alpha_{r}}^{(1)}\right)-\left(\bar{\partial} \chi-\chi \bar{\partial} \varphi_{2}+\left(\frac{1}{2 \lambda_{1}^{2}}-1\right) \frac{\chi^{2} \bar{\partial} \psi}{\Delta} e^{-\varphi_{2}}\right) e^{-\frac{1}{2} R} E_{-\alpha_{1}}^{(0)} \\
& -\frac{\bar{\partial} \psi}{\Delta} e^{\frac{1}{2} R-\varphi_{2}} E_{\alpha_{1}}^{(0)}-\bar{\partial} \nu \hat{c}-\bar{\partial} \eta \hat{d}-\sum_{i=2}^{r} \bar{\partial} \varphi_{i} h_{i}^{(0)}-\frac{\chi \bar{\partial} \psi}{\Delta} e^{-\varphi_{2}} \sum_{j=2}^{r}\left(\frac{r+1-j}{r}\right) h_{j}^{(0)}
\end{aligned}
$$

leading to the following equations of motion

$$
\begin{gathered}
\partial \bar{\partial} \eta=0, \quad \partial \bar{\partial} \nu=\mu^{2} e^{\varphi_{r}-\eta}\left(e^{\varphi_{2}}+\psi \chi\right) \\
\partial\left(\frac{e^{-\varphi_{2}} \bar{\partial} \psi}{\Delta}\right)+\left(\frac{r+1}{2 r}\right) \frac{\psi e^{-2 \varphi_{2}} \partial \chi \bar{\partial} \psi}{\Delta^{2}}+\mu^{2} e^{\varphi_{r}-\eta} \psi=0
\end{gathered}
$$




$$
\begin{gathered}
\bar{\partial}\left(\frac{e^{-\varphi_{2}} \partial \chi}{\Delta}\right)+\left(\frac{r+1}{2 r}\right) \frac{\chi e^{-2 \varphi_{2}} \partial \chi \bar{\partial} \psi}{\Delta^{2}}+\mu^{2} e^{\varphi_{r}-\eta} \chi=0 \\
\partial \bar{\partial} \varphi_{i}+\left(\frac{r+1-i}{r}\right) \frac{\partial \chi \bar{\partial} \psi e^{-\varphi_{2}}}{\Delta^{2}} \\
+\mu^{2} e^{\varphi_{2}+\varphi_{r}-\eta}\left(1+\left(\frac{i-1}{r}\right) \psi \chi e^{-\varphi_{2}}\right)-\mu^{2} e^{-\sum_{j=2}^{r} K_{i j} \varphi_{j}}=0
\end{gathered}
$$

$i=2, \cdots r$, where we have normalized $\alpha^{2}=2$.

The equations of motion (3.6)-(3.9) can be derived from the action

$$
\begin{aligned}
S_{e f f} & =-\frac{k}{4 \pi} \int d^{2} x\left(\sum_{i, j=2}^{r} K_{i, j} \partial \varphi_{i} \bar{\partial} \varphi_{j}+\partial \nu \bar{\partial} \eta+\partial \eta \bar{\partial} \nu\right. \\
& \left.+\frac{2 e^{-\varphi_{2}} \partial \chi \bar{\partial} \psi}{\Delta}-2 \mu^{2}\left(\sum_{i=2}^{r} e^{-\sum_{j=2}^{r} K_{i, j} \varphi_{j}}+e^{\varphi_{r}+\varphi_{2}-\eta}\left(1+\psi \chi e^{-\varphi_{2}}\right)\right)\right)
\end{aligned}
$$

The fundamental Poisson bracket relation (FPR) can be derived for the $A_{x}=A-\bar{A}$ component of the two dimensional gauge connection. It relates the cannonical structure derived from the action (3.10) and a Lie algebraic structure by the classical $r$ matrix. Explicit construction of the FPR is given in ref. [14].

The model is invariant under conformal transformations, i.e. $z \rightarrow f(z)$ e $\bar{z} \rightarrow \bar{f}(\bar{z})$, where $(f, \bar{f})$ are analytic functions and the fields transform as

$$
\begin{aligned}
& \chi(z, \bar{z}) \rightarrow\left(\bar{f}^{\prime}\right)^{\frac{1-r}{2}} \chi(f, \bar{f}), \quad \psi(z, \bar{z}) \rightarrow\left(f^{\prime}\right)^{\frac{1-r}{2}} \psi(f, \bar{f}), \\
& \eta(z, \bar{z}) \rightarrow \eta(f, \bar{f})-r \ln \left(f^{\prime}(z) \bar{f}^{\prime}(\bar{z})\right) \\
& \varphi_{i}(z, \bar{z}) \rightarrow \varphi_{i}(f, \bar{f})-\frac{(i-1)(r-i+1)}{2} \ln \left(f^{\prime}(z) \bar{f}^{\prime}(\bar{z})\right), \quad i=2, \cdots r \\
& \nu(z, \bar{z}) \rightarrow \nu(f, \bar{f})+\delta \ln \left(f^{\prime}(z) \bar{f}^{\prime}(\bar{z})\right)
\end{aligned}
$$

where $\delta$ is arbitrary. The infinite dimensional conformal symmetry (3.11) is generated by the chiral componentes of the improved stress energy momentum tensor

$$
\begin{aligned}
T(z) & =-\frac{k}{4 \pi}\left(\frac{1}{2} K_{i, j} \partial \varphi_{i} \partial \varphi_{j}+\frac{\partial \psi \partial \chi}{\Delta} e^{-\varphi_{2}}+\partial \eta \partial \nu\right. \\
& \left.+\frac{(r-1)}{2} \partial\left(\frac{\psi \partial \chi}{\Delta} e^{-\varphi_{2}}\right)+\partial^{2}\left(\sum_{i=2}^{r} \varphi_{i}+r \nu\right)\right)
\end{aligned}
$$

and the same for $\bar{T}$ obtained by $\partial \rightarrow \bar{\partial}, \psi \rightarrow \chi$.

\section{Dressing Transformation, Vertex Operators and De- generate Physical States}




\subsection{Dressing}

The conformal invariance may be broken by taking a particular solution of the first eqn. of motion in (3.6) namely $\eta=$ const. Equations (3.7)-(3.9) do not depend on $\nu$ and can be solved independently. The set of equations (3.7)-(3.9) with $\eta=$ const defines the affine NA Toda model and their zero curvature representation is given by (3.4) and (3.5) with $\eta=$ const.

A well established method for determining soliton solutions is provided by the dressing of a vacuum into a non trivial solution by gauge transformation [13]. The zero curvature condition implies pure gauge connections, $A=-\partial T T^{-1}$ and $\bar{A}=-\bar{\partial} T T^{-1}$. Suppose there exist a vacuum solution satisfying

$$
\bar{\partial} B_{v a c} B_{v a c}^{-1}=\mu^{2} z \hat{c}, \quad B_{v a c} \epsilon^{-} B_{v a c}^{-1}=\epsilon^{-}
$$

leading to

$$
A_{v a c}=\epsilon^{-}, \quad \bar{A}_{v a c}=-\epsilon^{+}-\mu^{2} z \hat{c} .
$$

where $\left[\epsilon^{+}, \epsilon^{-}\right]=\mu^{2} \hat{c}$. The solution for $A_{v a c}=-\partial T_{0} T_{0}^{-1}$ and $\bar{A}_{v a c}=-\bar{\partial} T_{0} T_{0}^{-1}$ is therefore given by

$$
T_{0}=\exp \left(-z \epsilon^{-}\right) \exp \left(\bar{z} \epsilon^{+}\right)
$$

The dressing method is based on the assumption of the existence of two gauge transformations generated by $\Theta^{ \pm}$, mapping the vacuum into non trivial configuration, i.e.

$$
A=\Theta^{ \pm} A_{v a c}\left(\Theta^{ \pm}\right)^{-1}-\partial \Theta^{ \pm}\left(\Theta^{ \pm}\right)^{-1}
$$

and

$$
\bar{A}=\Theta^{ \pm} \bar{A}_{v a c}\left(\Theta^{ \pm}\right)^{-1}-\bar{\partial} \Theta^{ \pm}\left(\Theta^{ \pm}\right)^{-1}
$$

or

$$
B \epsilon^{-} B^{-1}=\Theta^{ \pm} \epsilon^{-}\left(\Theta^{ \pm}\right)^{-1}-\partial \Theta^{ \pm}\left(\Theta^{ \pm}\right)^{-1}
$$

and

$$
-\epsilon^{+}-\bar{\partial} B B^{-1}=\Theta^{ \pm}\left(-\epsilon^{+}-\mu^{2} z \hat{c}\right)\left(\Theta^{ \pm}\right)^{-1}-\bar{\partial} \Theta^{ \pm}\left(\Theta^{ \pm}\right)^{-1}
$$

As a consequence we relate

$$
\Theta^{+} T_{0}=\Theta^{-} T_{0} g
$$

where $g$ is an arbitrary constant group element. We suppose that $\Theta^{ \pm}$are group elements of the form

$$
\Theta^{-}=e^{t(0)} e^{t(-1)} e^{t(-2)} \cdots, \quad \Theta^{+}=e^{v(0)} e^{v(1)} e^{v(2)} \ldots
$$

where $t^{(-i)}, v^{(i)}$ are linear combinations of grade $(-i)$ and $(i)$ generators respectively $(i=$ $0,1, \cdots)$. In considering $\Theta^{-}$the zero grade component of $(4.6$ admits solution

$$
t(0)=e^{H(\bar{z})}
$$

For $\Theta^{+}$the zero grade of equation (4.7) admits

$$
e^{v(0)}=B e^{G(z)-\mu^{2} z \bar{z} \hat{c}}
$$


where $H(\bar{z}), G(z) \in \mathcal{G}_{0}^{0}$, i.e. commute with both $\epsilon^{ \pm}$. From (4.7) for $\Theta^{-}$we get for the zero grade

$$
\bar{\partial} B B^{-1}=e^{H(\bar{z})}\left[t(-1), \epsilon^{+}\right] e^{-H(\bar{z})}+\mu^{2} z \hat{c}+\left(\bar{\partial} e^{H(\bar{z})}\right) e^{-H(\bar{z})}
$$

From (4.6) for $\Theta^{+}$we obtain by comparing the zero grade

$$
0=e^{v(0)}\left[v(1), \epsilon^{-}\right] e^{-v(0)}-\partial e^{v(0)} e^{-v(0)}
$$

Inserting (4.11) in (4.13) we get

$$
B^{-1} \partial B-\mu^{2} \bar{z} \hat{c}=e^{G(z)}\left[v(1), \epsilon^{-}\right] e^{-G(z)}-B^{-1}\left(\partial e^{G(z)}\right) e^{-G(z)} B
$$

Multipling (4.12) and (4.14) by an element of $\mathcal{G}_{0}^{0}$ and taking the trace we find that the choice $H(\bar{z})=G(z)=0$ implies the subsidiary conditions

$$
\operatorname{Tr}\left(B^{-1} \partial B \mathcal{G}_{0}^{0}\right)=\operatorname{Tr}\left(\bar{\partial} B B^{-1} \mathcal{G}_{0}^{0}\right)=0 .
$$

and in eqns. (3.2). From eqn. (4.8) we find

$$
\ldots e^{-t(-2)} e^{-t(-1)} B e^{-\mu^{2} \bar{z} z \hat{c}} e^{v(1)} e^{v(2)} \ldots=T_{0} g T_{0}^{-1} .
$$

hence,

$$
<\lambda^{\prime}\left|B \exp \left(-\mu^{2} z \bar{z} \hat{c}\right)\right| \lambda>=<\lambda^{\prime}\left|T_{0} g T_{0}^{-1}\right| \lambda>.
$$

where $\mid \lambda>$ and $<\lambda^{\prime} \mid$ are annihilated by $\mathcal{G}_{>}$and $\mathcal{G}_{<}$respectively. Explicit space time dependence for fields $\in \mathcal{G}_{0} / \mathcal{G}_{0}^{0}$ is given by choosing specific matrix elements, defining the so called tau functions,

$$
\begin{aligned}
\tau_{0} \equiv e^{\nu-\mu^{2} z \bar{z}} & =<\lambda_{0}\left|T_{0} g T_{0}^{-1}\right| \lambda_{0}> \\
\tau_{R} \equiv e^{\frac{r R}{r+1}+\nu-\mu^{2} z \bar{z}} & =<\lambda_{1}\left|T_{0} g T_{0}^{-1}\right| \lambda_{1}>, \\
\tau_{j} \equiv e^{\lambda_{1} \cdot \lambda_{j} R+\varphi_{j}+\nu-\mu^{2} z \bar{z}} & =<\lambda_{j}\left|T_{0} g T_{0}^{-1}\right| \lambda_{j}>, \quad j=2, \cdots r \\
\tau_{\psi} \equiv e^{\frac{1}{2} \frac{r-1}{r+1} R+\nu-\mu^{2} z \bar{z}} \psi & =<\lambda_{1}\left|T_{0} g T_{0}^{-1} E_{-\alpha_{1}}^{(0)}\right| \lambda_{1}>, \\
\tau_{\chi} \equiv e^{\frac{1}{2} \frac{r-1}{r+1} R+\nu-\mu^{2} z \bar{z}} \chi & <\lambda_{1}\left|E_{\alpha_{1}}^{(0)} T_{0} g T_{0}^{-1}\right| \lambda_{1}>
\end{aligned}
$$

where for $S L(r+1), \lambda_{1} \cdot \lambda_{j}=\frac{r+1-j}{r+1}$.

\subsection{Vertex Operators}

Suppose we now write the constant group element $g$ in (4.8) as

$$
g=\exp [F(\gamma)]
$$

where $\gamma$ is complex parameter and choose $F(\gamma)$ to be an eigenstate of $\epsilon^{ \pm}$, i.e.

$$
\left[\epsilon^{ \pm}, F(\gamma)\right]=f^{ \pm}(\gamma) F(\gamma)
$$

where $f^{ \pm}$are specific functions of $\gamma$. It therefore follows that,

$$
T_{0} g T_{0}^{-1}=\exp \{\rho(\gamma) F(\gamma)\}
$$


where

$$
\rho=\exp \left\{-z f^{-}(\gamma)+\bar{z} f^{+}(\gamma)\right\}
$$

For more general cases where

$$
g=\exp \left[F_{1}\left(\gamma_{1}\right)\right] \exp \left[F_{2}\left(\gamma_{2}\right)\right] \ldots \exp \left[F_{N}\left(\gamma_{N}\right)\right]
$$

with

$$
\left[\epsilon^{ \pm}, F_{i}\left(\gamma_{i}\right)\right]=f_{i}^{ \pm}\left(\gamma_{i}\right) F_{i}\left(\gamma_{i}\right)
$$

we find

$$
T_{0} g T_{0}^{-1}=\exp \left[\rho_{1}\left(\gamma_{1}\right) F_{1}\left(\gamma_{1}\right)\right] \exp \left[\rho_{2}\left(\gamma_{2}\right) F_{2}\left(\gamma_{2}\right)\right] \ldots \exp \left[\rho_{N}\left(\gamma_{N}\right) F_{N}\left(\gamma_{N}\right)\right]
$$

where

$$
\rho_{i}\left(\gamma_{i}\right)=\exp \left[-z f_{i}^{-}\left(\gamma_{i}\right)+\bar{z} f_{i}^{+}\left(\gamma_{i}\right)\right] .
$$

For the $A_{r}$ case, it is possible to show that $F^{2}=0$ since $F\left(\gamma_{i}\right)$ is constructed in terms of vertex operators 23]. In our specific model, the gradation interpolates between the homogeneous and the principal gradations given by $Q=r D+\sum_{i=2}^{r} \frac{2 \lambda_{i} \cdot H^{(0)}}{\alpha_{i}^{2}}$. The constant grade \pm 1 generators are

$$
\epsilon^{+}=\mu\left(\sum_{i=2}^{r} E_{\alpha_{i}}^{(0)}+E_{-\left(\alpha_{2}+\ldots+\alpha_{r}\right)}^{(1)}\right), \quad \epsilon^{-}=\mu\left(\sum_{i=2}^{r} E_{-\alpha_{i}}^{(0)}+E_{\alpha_{2}+\ldots+\alpha_{r}}^{(-1)}\right),
$$

and their eigenstates are known to be [22], 23]

$$
\begin{aligned}
F_{1, j}^{ \pm}(\gamma)=\sum_{n=-\infty}^{\infty} \gamma^{-r n} \sum_{p=0}^{r-1} w^{ \pm p j} \gamma^{\mp p} E_{ \pm\left(\alpha_{1}+\ldots+\alpha_{(p+1)}\right)}^{(n)} \\
F_{a, j}=\frac{\hat{c}}{\left(w^{a}-1\right)}+\sum_{n \in Z} \gamma^{-r n} \sum_{i=1}^{r-1} h_{i+1}^{(n)} \sum_{p=1}^{i} w^{a(i-p)} \\
+\sum_{b=1}^{r-1} \sum_{n \in Z} w^{b j} \gamma^{-(b+n r)}\left(\sum_{i=1}^{r-b} w^{a(i-1)} E_{\alpha_{i+1}+\alpha_{i+2}+\cdots+\alpha_{i+b}}^{(n)}\right. \\
\left.+\sum_{i=1}^{b} w^{a(i+r-1-b)} E_{-\left(\alpha_{i+1}+\alpha_{i+2}+\cdots+\alpha_{i+r-b}\right)}^{(n+1)}\right)
\end{aligned}
$$

where $j=1, \ldots, r, a=1, \ldots, r-1$ and $w=\exp \left(\frac{2 \pi i}{r}\right)$. Their eigenvalues are obtained from

$$
\begin{gathered}
{\left[\epsilon^{ \pm}, F_{1, j}^{s}(\gamma)\right]=-s \mu w^{\mp j} \gamma^{ \pm} F_{1, j}^{s}(\gamma), \quad s= \pm} \\
{\left[\epsilon^{ \pm}, F_{a, j}(\gamma)\right]=\mu w^{\mp j}\left(w^{ \pm a}-1\right) \gamma^{ \pm} F_{a, j}(\gamma) .}
\end{gathered}
$$

Although there are $r(r+1)$ vertices we can classify them into subsets such that all vertices within a subset are equivalent in the sense that they all provide the same solution 
by redefinition of the spectral parameter. In fact, such equivalence is established directly from the form of the eigenstates. It then follows

$$
\begin{aligned}
& F_{1, r}^{ \pm}\left(\gamma=\widetilde{\gamma} w^{-j}\right)=F_{1, j}^{ \pm}(\gamma=\widetilde{\gamma}), \\
& F_{a, r}\left(\gamma=\widetilde{\gamma} w^{-j}\right)=F_{a, j}(\gamma=\widetilde{\gamma}) .
\end{aligned}
$$

For convenience we shall be considering solutions corresponding to one representative of each subset, namely, $F_{1, r}^{ \pm}, F_{d, r}, d=1, \cdots r-1$. For $\gamma^{*}=\gamma^{-1}$ one can find,

$$
F_{1, j}^{+\dagger}(\gamma)=F_{1, j}^{-}(\gamma), \quad F_{a, j}^{\dagger}(\gamma)=F_{r-a,(j-a) \bmod r}(\gamma)
$$

\subsection{Degenerate Physical States - The Structure of Solutions}

In this section we shall classify the soliton solutions, and discuss their properties in terms of the algebraic structure of the $r+1$ vertex operators: $F_{1, r}^{ \pm}, F_{d, r}, d=1, \cdots, r-1$.

Appart from the vacuum solution, characterized by zero energy and momentum, the basic soliton solutions are called 1-soliton ( 1-antisoliton ), the scattering of two 1-solitons results in a 2-soliton solution and so on. There are also bound states of two 1-soliton solutions, whose basic property is that their energy is smaller than the sum of energies corresponding to two 1-soliton solutions.

A solution constructed with a single vertex $g=e^{F}$ where $F=F_{1, r}^{-}$or $F_{1, r}^{+}$is a vacuum solution, since they contain only step operators and annihilate the diagonal matrix elements in (4.18). Those matrix elements define the spectra (i.e., mass, energy, momentum, electric charge). In the first case the only nontrivial tau function is

$$
\tau_{\chi}=a \exp \left[-\frac{\mu z}{\gamma}+\mu \gamma \bar{z}\right]
$$

where $(a, \gamma)$ are complex constants. The other tau functions vanish identically, i.e.

$$
\tau_{0}=\tau_{R}=\tau_{\psi}=\tau_{j}=0
$$

This implies that the field $\chi \neq 0$ and $\psi=0$. Since the stress energy momentum depend upon the product $\psi \chi$ and its derivatives, such solution corresponds to energy and momentum $E=$ $P=0$. Analogously for $F_{1, r}^{+}$where $\psi \rightarrow \chi$ and similarly for the sum $\sum_{i=1}^{N} a_{i} F_{1, r}^{s}\left(\gamma_{i}\right), s= \pm 1$. In other words, $N$ vertices $F_{1, r}^{-}$or $F_{1, r}^{+}$result in the physical vacuum (where $N$ is arbitrary).

There are two basic 1-soliton solutions. They are provided by the constant group elements

$$
g=\exp \left[c F_{d, r}(\gamma)\right]
$$

or

$$
g=\exp \left[a F_{1, r}^{-}\left(\gamma_{1}\right)\right] \exp \left[b F_{1, r}^{+}\left(\gamma_{2}\right)\right]
$$

The first provide electric charge $Q_{e l}=0$, since the structure of $F_{d, r}$ in eqns. (4.18) leads to $R=0\left(F_{d, r}\right.$ does not contain $\left.h_{1}^{(0)}\right)$, while in the second $Q_{e l} \neq 0$. In this sense one can say 
that $F_{d, r}$ are neutral vertices while both $F_{1, r}^{-}, F_{1, r}^{+}$are charged vertices. It will be shown in the next section that (4.37) provides the solution of the $A_{r-1}^{(1)}$ abelian affine Toda model.

The solutions obtained from

$$
g=\exp \left[c F_{d, r}(\gamma)\right] \Pi_{i=1}^{N} \exp \left[a_{i} F_{1, r}^{s}\left(\gamma_{i}\right)\right]
$$

and

$$
g=\exp \left[a F_{1, r}^{-}\left(\gamma_{1}\right)\right] \exp \left[b F_{1, r}^{+}\left(\gamma_{2}\right)\right] \exp \left[c F_{1, r}^{s}\left(\gamma_{3}\right)\right]
$$

where $s=+1$ or $s=-1$ correspond to the same energy and momentum as those obtained from (4.37) and (4.38) respectively. However their explicit form ( $\tau$-functions ) differ and may involve more parameters (see Sect. 5).

There are three basic 2-soliton solutions characterized by

$$
\begin{gathered}
g=\exp \left[c_{1} F_{d_{1}, r}\left(\gamma_{1}\right)\right] \exp \left[c_{2} F_{d_{2}, r}\left(\gamma_{2}\right)\right] \\
g=\exp \left[a F_{1, r}^{-}\left(\gamma_{1}\right)\right] \exp \left[b F_{1, r}^{+}\left(\gamma_{2}\right)\right] \exp \left[c F_{d, r}\left(\gamma_{3}\right)\right] \\
g=\exp \left[a F_{1, r}^{-}\left(\gamma_{1}\right)\right] \exp \left[b F_{1, r}^{+}\left(\gamma_{2}\right)\right] \exp \left[c F_{1, r}^{-}\left(\gamma_{3}\right)\right] \exp \left[d F_{1, r}^{+}\left(\gamma_{4}\right)\right]
\end{gathered}
$$

It will be shown that the respective energies correspond to the sum of the 1-soliton individual energy.

The solution corresponding to (4.41) is the 2-soliton of the $A_{r-1}^{(1)}$ abelian affine Toda model. If we now take

$$
g=\exp \left[c_{1} F_{d_{1}, r}\left(\gamma_{1}\right)\right] \exp \left[c_{2} F_{d_{2}, r}\left(\gamma_{2}\right)\right] \Pi_{i=1}^{N} \exp \left[a_{i} F_{1, r}^{s}\left(\gamma_{i}\right)\right]
$$

$s= \pm 1$, the corresponding solutions will have the same energy and momentum as those obtained from (4.41) and are also 2-soliton solutions.

We have three basic bound states solutions. As in the 2-soliton solutions they arise from (4.41), (4.42) and (4.43), but the specific choice of the parameters $\gamma$ is different.

\section{1-Soliton Solutions}

\subsection{Neutral 1-soliton}

Our model (1.1) has two basic independent 1-soliton solutions. The first one is obtained by taking the constant group element $g$ as

$$
g=\exp \left[c F_{d, r}(\gamma)\right]
$$

where $F_{d, r}$ is given in (4.29), $c, \gamma$ are complex constants and $d=1, \cdots, r-1$ describe the different species of the soliton. The corresponding solution solution obtained from (4.18) is

$$
\tau_{0}=\tau_{R}=1+e^{\zeta+H}, \quad \tau_{\psi}=\tau_{\chi}=0 ; \quad \tau_{j}=1+w^{d(j-1)} e^{\zeta+H}, \quad j=2, \ldots, r
$$

where

$$
H=2 \mu(-1)^{n} \sin \left(\frac{\pi d}{r}\right) \cosh (S)(x-t \tanh (S)), \quad n \in Z, \quad S \in R
$$


$w=\exp \left(\frac{2 \pi i}{r}\right)$ and $\zeta=\zeta_{R}+i \zeta_{I}$ are related to the specific parametrization

$$
\gamma=i(-1)^{n} w^{-\frac{d}{2}} e^{S}, \quad c=\left(w^{d}-1\right) e^{\zeta}
$$

This choice of $\gamma$ is such that the solution will have real energy and momentum. The parameter $S$ is the rapidity $|v|=\tanh (S)$. The real part of $\zeta=\zeta_{R}$ corresponds to shifts in space time and its imaginary part $\zeta_{I}$ is in general associated to the topological charge.

Since the nonlocal field $R$ is constant it follows that $Q_{e l}=0$. It is in this sense that one can say that $F_{d, r}$ are neutral vertices. The topological charge is determined in terms of non trivial zeros of the potential (see (2.25)) by $\varphi_{j}$, i.e.

$$
Q_{j}^{t o p}=\frac{1}{2 \pi i} \int_{-\infty}^{\infty} \partial_{x} \varphi_{j} d x=(j-1) Q_{m a g}, \quad j=2, \cdots r
$$

where $Q_{\text {mag }}= \pm\left.\frac{1}{r}(d)\right|_{\text {mod } r}$

The mass is obtained from $\tau_{0}$ as described in the appendix D

$$
M=\frac{E}{\cosh (S)}=\frac{P}{\sinh S}=\frac{2 k \mu r}{\pi} \sin \left(\frac{\pi d}{r}\right)
$$

corresponding to the mass formula of the abelian affine Toda model $A_{r-1}^{(1)}$ [20].

An important remark to be pointed out is the fact that degenerate solutions can be obtained by replacing the constant group element $g$ in eqn. (5.1) by

$$
g=\exp \left[c F_{d, r}(\gamma)\right] \Pi_{i=1}^{N} \exp \left[a_{i} F_{1, r}^{s}\left(\gamma_{i}\right)\right]
$$

with arbitrary $N$. Since $F_{1, r}^{ \pm}$only contain step operators, the resulting solution yields the same spectra of mass, electric and topological charges. For instance, the group element in (5.7) with $s=-1$ produces the same tau functions as in (5.2), the only exception being one nonvanishing tau function,

$$
\tau_{\chi}=\sum_{i=1}^{N} a_{i} \exp \left[-\frac{\mu z}{\gamma_{i}}+\mu \gamma_{i} \bar{z}\right], \quad \tau_{\psi}=0
$$

Consequently, the field $\chi$ is different from zero but $\psi=0$. Since the energy momentum tensor depends explicitly on the product $\psi \chi$ and on its derivatives, the solutions defined by $g$ given in (5.1) and in (5.7) with $s=-1$ are degenerate. Similarly, one can check that electric and topological charges remain unchanged. The same is true with the solution obtained from $g$ in (5.7) with $s=+1$ interchanging $\psi$ by $\chi$.

\subsection{Charged 1-Soliton Solution}

In a our previous paper [9], electrically charged topological 1-soliton solution have been constructed using the method of Backlund transformations. Here, we will employ the dressing method with $g$ chosen as

$$
g=\exp \left[a F_{1, r}^{-}\left(\gamma_{1}\right)\right] \exp \left[b F_{1, r}^{+}\left(\gamma_{2}\right)\right]
$$


where $F_{1, r}^{ \pm}$are given in (4.28) and $a, b, \gamma_{1}$ and $\gamma_{2}$ are complex constants. From (4.18) we obtain the solution

$$
\begin{aligned}
\tau_{0} & =1+e^{\zeta+2 F}, \quad \tau_{R}=1+\left(\Gamma_{1}\right)^{-r} e^{\zeta+2 F} \\
\tau_{j} & =1+\left(\Gamma_{1}\right)^{j-r-1} e^{\zeta+2 F}, \quad \tau_{\chi}=\Gamma_{2} e^{\theta+i G} e^{\frac{\zeta}{2}}, \quad \tau_{\psi}=\Gamma_{2} e^{-(\theta+i G)} e^{\frac{\zeta}{2}}
\end{aligned}
$$

$j=2, \cdots r$, where

$$
\begin{gathered}
\Gamma_{1}=-\exp (-2 i \bar{a})=\frac{\gamma_{1}}{\gamma_{2}}, \quad \Gamma_{2}=\sqrt{\left(1-\Gamma_{1}\right)\left(\left(\Gamma_{1}\right)^{-r}-1\right)} . \\
F=\mu \cos (\bar{a}) \cosh (\bar{b})(x-t \tanh (\bar{b})), \quad G=\mu \sin (\bar{a}) \sinh (\bar{b})(t \operatorname{coth}(\bar{b})-x)
\end{gathered}
$$

$\bar{a}, \bar{b}$ are real parameters, $\Theta, \zeta$ are complex parameters. They correspond to a specific parametrization

$$
\begin{gathered}
\gamma_{1}=-\exp (\bar{b}-i \bar{a}), \quad \gamma_{2}=\exp (\bar{b}+i \bar{a}) \\
a=\sqrt{\frac{\left(1-\gamma_{1,2}\right)\left(1-\left(\gamma_{1,2}\right)^{r}\right)}{\left(\gamma_{1,2}\right)^{r}}} \exp \left(\Theta+\frac{1}{2} \zeta\right)
\end{gathered}
$$

and

$$
b=\sqrt{\frac{\left(1-\gamma_{1,2}\right)\left(1-\left(\gamma_{1,2}\right)^{r}\right)}{\left(\gamma_{1,2}\right)^{r}}} \exp \left(-\Theta+\frac{1}{2} \zeta\right),
$$

where $\gamma_{i, j}=\frac{\gamma_{i}}{\gamma_{j}}$. The choice of $\left(\gamma_{1}, \gamma_{2}\right)$ is such that ensures real energy and momentum.

The asymptotics of the field $R$ lead to the electric charge

$$
Q_{e l}=-\frac{2 k r}{\pi}\left[\operatorname{sign}(\cos (\bar{a}))\left(\frac{\pi}{2} \operatorname{sign}(\bar{a})-\bar{a}\right)-\left(n_{1}^{+}-n_{1}^{-}\right) \frac{\pi}{r}\right], \quad n_{1}^{ \pm} \in Z .
$$

and $Q_{e l}=0$ if $\cos (\bar{a})=0$. The topological charge $Q_{j}^{t o p}=(j-1) Q_{\text {mag }}$ is given in terms of the asymtoptics of the fields $\varphi_{j}(2.25)$,

$$
Q_{j}^{\text {top }}=\frac{(j-1)}{r}\left(n^{+}-n_{-}\right)_{\bmod r}, \quad n^{ \pm} \in Z
$$

Using again the argument explained in the appendix D, we evaluate the mass of the solution, yielding

$$
M=\frac{E}{\cosh (\bar{b})}=\frac{P}{\sinh (\bar{b})}=\frac{2 k \mu r}{\pi}|\cos (\bar{a})|
$$

The same solution was also found using first order differential equations obtained from Backlund transformation in ref. [9]. Following the discussion of Sect. 4.3, we shall show that solution associated with

$$
g=\exp \left[a F_{1, r}^{-}\left(\gamma_{1}\right)\right] \exp \left[b F_{1, r}^{+}\left(\gamma_{2}\right)\right] \exp \left[c F_{1, r}^{s}\left(\gamma_{3}\right)\right]
$$


will have the same energy and momentum as the one derived above. The general solution can be calculated yielding, for $s=-1$

$$
\begin{aligned}
\tau_{0} & =1+a b \frac{\left(\gamma_{1,2}\right)^{r} e^{F_{a}+F_{b}}}{\left(1-\gamma_{1,2}\right)\left(1-\left(\gamma_{1,2}\right)^{r}\right)}+b c \frac{\left(\gamma_{3,2}\right)^{r} e^{F_{b}+F_{c}}}{\left(1-\gamma_{3,2}\right)\left(1-\left(\gamma_{3,2}\right)^{r}\right)} \\
\tau_{R} & =1+a b \frac{e^{F_{a}+F_{b}}}{\left(1-\gamma_{1,2}\right)\left(1-\left(\gamma_{1,2}\right)^{r}\right)}+b c \frac{e^{F_{b}+F_{c}}}{\left(1-\gamma_{3,2}\right)\left(1-\left(\gamma_{3,2}\right)^{r}\right)} \\
\tau_{j} & =1+a b \frac{\left(\gamma_{1,2}\right)^{j-1} e^{F_{a}+F_{b}}}{\left(1-\gamma_{1,2}\right)\left(1-\left(\gamma_{1,2}\right)^{r}\right)}+b c \frac{\left(\gamma_{3,2}\right)^{j-1} e^{F_{b}+F_{c}}}{\left(1-\gamma_{3,2}\right)\left(1-\left(\gamma_{3,2}\right)^{r}\right)} \\
\tau_{\chi} & =a e^{F_{a}}+c e^{F_{c}}, \quad \tau_{\psi}=b e^{F_{b}}
\end{aligned}
$$

where

$$
\gamma_{1}=e^{\hat{a}+i \bar{a}}, \quad \gamma_{2}=e^{\hat{b}+i \bar{b}}, \quad \gamma_{3}=e^{\hat{c}+i \bar{c}}
$$

$\bar{a}, \hat{a}, \bar{b}, \hat{b}, \bar{c}, \hat{c}$ are real numbers and

$$
\begin{aligned}
& F_{a}=\mu t(\cos (\bar{a}) \sinh (\hat{a})+i \sin (\bar{a}) \cosh (\hat{a}))-\mu x(\cos (\bar{a}) \cosh (\hat{a})+i \sin (\bar{a}) \sinh (\hat{a})) \\
& F_{c}=F_{a}(\bar{a} \rightarrow \bar{c}, \hat{a} \rightarrow \hat{c}), \quad F_{b}=-F_{a}(\bar{a} \rightarrow \bar{b}, \hat{a} \rightarrow \hat{b})
\end{aligned}
$$

Following appendix D, the energy and the momentum for the case $\lim _{x \rightarrow+\infty}\left(F_{a}+F_{b}\right)=\lim _{x \rightarrow-\infty}\left(F_{b}+\right.$ $\left.F_{c}\right)=+\infty$ are given as,

$$
\begin{aligned}
E & =-\frac{k \mu r}{\pi}(\cos (\bar{a}) \cosh (\hat{a})+i \sin (\bar{a}) \sinh (\hat{a})-\cos (\bar{c}) \cosh (\hat{c})-i \sin (\bar{c}) \sinh (\hat{c})) \\
P & =\frac{k \mu r}{\pi}(\cos (\bar{a}) \sinh (\hat{a})+i \sin (\bar{a}) \cosh (\hat{a})-\cos (\bar{c}) \sinh (\hat{c})-i \sin (\bar{c}) \cosh (\hat{c}))
\end{aligned}
$$

The reality of the energy and momentum imply

$$
\sin (\bar{a}) \sinh (\hat{a})=\sin (\bar{c}) \sinh (\hat{c}), \quad \sin (\bar{a}) \cosh (\hat{a})=\sin (\bar{c}) \cosh (\hat{c})
$$

leading to

$$
\hat{a}=\hat{c}, \quad \sin (\bar{a})=\sin (\bar{c}), \quad \cos (\bar{a})= \pm \cos (\bar{c}) .
$$

The choice $\cos (\bar{a})=\cos (\bar{c})$ gives zero mass configuration. The other choice results in a perfect agreement with the mass formula already obtained with $c=0$

$$
M=\frac{E}{\cosh (\hat{a})}=\frac{P}{\sinh (\hat{a})}=\frac{2 k \mu r}{\pi}|\cos (\bar{a})|
$$

Taking $\bar{c}=\pi \operatorname{sign}(\bar{a})-\bar{a}$ we get the same electric charge $Q_{e l}$ in (5.16). The other cases with different asymptotic behaviour lead to the same results. The solution with $s=+1$ also gives the same conclusion. 


\section{Two Soliton Solutions and Time Delays}

\subsection{Two Soliton Solutions}

The 2-soliton solutions represent the scattering of two 1-soliton solutions. These may be composed of neutral or charged vertices given by products of exponentials associated to $F_{d, r}$ and $\left(F_{1, r}^{+}, F_{1, r}^{-}\right)$. Combining the vertices we can obtain three different classes of 2-solitons:

- neutral-neutral (abelian) $\left(F_{d, r}\right) \otimes\left(F_{d, r}\right)$

- neutral-charged $\left(F_{d, r}\right) \otimes\left(F_{1, r}^{+}, F_{1, r}^{-}\right)$

- charged-charged $\left(F_{1, r}^{+}, F_{1, r}^{-}\right) \otimes\left(F_{1, r}^{+}, F_{1, r}^{-}\right)$

The same vertices also provide bound states solutions. These differ by the specific parametrization of the spectral parameters. We will discuss, in this section the construction of the neutral-charged case. The final result of the other two cases is shown in the appendix C. Finally we will discuss the spectrum of the three solutions.

Consider

$$
g=\exp \left[a F_{1, r}^{-}\left(\gamma_{1}\right)\right] \exp \left[b F_{1, r}^{+}\left(\gamma_{2}\right)\right] \exp \left[c F_{d, r}\left(\gamma_{3}\right)\right]
$$

where $a, b, c, \gamma_{i}$ are arbitrary complex constants, $d=1, \cdots, r-1$ correspond to the neutral specie. Details of the calculation of the tau functions (4.18) in terms of (6.1) are given in the appendix A. We therefore obtain, in general

$$
\tau=\sum_{n_{1}, n_{2}, n_{3}=0}^{1} A_{n_{1}, n_{2}, n_{3}}^{\tau} \exp \left[n_{1} A_{1}\left(\gamma_{1}\right)+n_{2} A_{2}\left(\gamma_{2}\right)+n_{3} A_{3}\left(\gamma_{3}\right)\right]
$$

where $\tau=\left(\tau_{0}, \tau_{R}, \tau_{j}, \tau_{\psi}, \tau_{\chi}\right), A_{n_{1}, n_{2}, n_{3}}^{\tau}$ are certain functions of $\gamma_{1}, \gamma_{2}, \gamma_{3}, a, b, c, A_{1}\left(\gamma_{1}\right)=$ $-\frac{\mu z}{\gamma_{1}}+\mu \gamma_{1} \bar{z}, A_{2}\left(\gamma_{2}\right)=A_{1}\left(-\gamma_{2}\right)$ and $A_{3}\left(\gamma_{3}\right)=-\frac{\mu z}{\gamma_{3}}\left(w^{-d}-1\right)+\mu \gamma_{3} \bar{z}\left(w^{d}-1\right)$. The tau functions given by (6.2) lead to solutions of the equations of motion (3.6)-(3.9), with $\eta=0$. However nothing is said about its solitonic nature, which can be 2 -soliton or a bound state. The key point is to choose the spectral parameters in an appropriate way to ensure the reality of the energy, momentum and electric charge (we emphasize that all solutions, including the 1-soliton were obtained following the same principle). For the neutral-charged case (6.2) we have

$$
\tau_{0}=1+A_{0,0,1}^{\tau_{0}} \exp \left(A_{3}\left(\gamma_{3}\right)\right)+A_{1,1,0}^{\tau_{0}} \exp \left(A_{1}\left(\gamma_{1}\right)+A_{2}\left(\gamma_{2}\right)\right)+A_{1,1,1}^{\tau_{0}} \exp \left(\sum_{i=1}^{3} A_{i}\left(\gamma_{i}\right)\right)
$$

and in general $(E, P)$ are proportional to linear combinations of $\partial_{x} A_{i}\left(\gamma_{i}\right)$ and $\partial_{t} A_{i}\left(\gamma_{i}\right)$, since they depend upon the logarithmic derivatives of $\tau_{0}$. The simplest choice is $A_{1}\left(\gamma_{1}\right)+A_{2}\left(\gamma_{2}\right)$ and $A_{3}\left(\gamma_{3}\right)$ to be real. One can see that this choice leads to $A_{3} \rightarrow H$ and $A_{1}+A_{2} \rightarrow 2 F$, where $H$ and $F$ are the functions associated, respectively to the 1-soliton, neutral and charged, expressions (5.3) and (5.12). Implying that such solution will have energy and momentum obtained by the combination of the corresponding 1-soliton values. In this case, the electric charge is already fixed to be real. The other nontrivial choice leads us to the bound state 
solution discussed in the next section. The explicit 2-soliton solution for all three cases are presented in the appendix C. The energy, momentum and masses of the three solutions are obtained according to eqn. (12.9) and (12.10)to be a surface term. They correspond to sums of quantities associated to the 1-soliton.

The electric charge being evaluated as asymptotics of the non local field $R$ is also a surface term. It can be verified that it is the sum of electric charges of 1-solitons. Clearly, the neutral-neutral is the abelian neutral 2-soliton and the charged-charged has two different contributions to $Q_{e l}$. The topological charge is also the sum of two 1-soliton quantities.

\subsection{Time Delays}

The two soliton solutions describe the scattering of two 1-soliton solutions. It is known that the effect of the scattering corresponds to a lateral displacement and associated time delay [17]. To make clear our discussion, consider 1-soliton solutions described by the field $\xi_{a}\left(x-x_{0 a}-v_{a} t\right)$. Suppose that there exist a 2-soliton solution and let $\xi_{a b}\left(x_{0 a}, x_{0 b}, v_{a}, v_{b}\right)$ be the associated field. Suppose also that, by taking $x-v_{a} t=$ const. we have

$$
\lim _{t \rightarrow \pm \infty} \xi_{a b}=K \xi_{a}\left(x-x_{0 a}-v_{a} t-x_{a}^{ \pm}\right)
$$

where $K$ is some complex constant. It means that we are following the 1 -soliton $a$. The lateral displacement is defined by $\Delta_{a}(x)=x_{a}^{+}-x_{a}^{-}$leading to the time delay

$$
\Delta_{a}(t)=-\frac{\Delta_{a}(x)}{v_{a}}
$$

In order to analyse the time delays for our 2-soliton solutions, note that the model has more than one field. Let us define a convenient combination of fields by

$$
\phi_{j}=\varphi_{j+1}-\varphi_{j}-\frac{R}{r+1}, \quad j=1, \cdots r
$$

$\varphi_{1}=\varphi_{r+1}=0$. There are three classes of 2-soliton solutions. We will analyse the neutralcharged with details and present the conclusions for the other two cases (the neutral-neutral (abelian ) is already shown in the literature [17]). The expression for $e^{\phi_{j}}$ is

$$
e^{\phi_{j}}=\frac{1+w^{d j} e^{\zeta_{1}+H}+\Gamma_{1}^{-r+j} e^{\zeta_{2}+2 F}+w^{d j} \Gamma_{1}^{-r+j} \Gamma_{N, c} e^{\zeta_{1}+\zeta_{2}} e^{H+2 F}}{1+w^{d(j-1)} e^{\zeta_{1}+H}+\Gamma_{1}^{-r+j-1} e^{\zeta_{2}+2 F}+w^{d(j-1)} \Gamma_{1}^{-r+j-1} \Gamma_{N, c} e^{\zeta_{1}+\zeta_{2}} e^{H+2 F}}
$$

where $H=2 \mu(-1)^{n} \sin \left(\frac{\pi d}{r}\right) \cosh (S)(x-t \tanh (S))$ and $F=\mu \cos (\bar{a}) \cosh (\bar{b})(x-t \tanh (\bar{b}))$ We take $\tanh (S)>0, \tanh (S)-\tanh (\bar{b})>0$ and follow the 1-soliton associated to $H$ :

$$
x-t \tanh (S)=\text { const }
$$

Substituting (6.8) in (6.7) we get for $\cos (\bar{a})>0$

$$
\lim _{t \rightarrow \infty} \exp \left[\phi_{j}\right]=\Gamma_{1}\left(\frac{1+e^{\zeta 1} w^{d j} \Gamma_{n, c} e^{H}}{1+e^{\zeta_{1}} w^{d(j-1)} \Gamma_{N, c} e^{H}}\right)
$$




$$
\lim _{t \rightarrow-\infty} \exp \left[\phi_{j}\right]=\left(\frac{1+e^{\zeta_{1}} w^{d j} e^{H}}{1+e^{\zeta_{1}} w^{d(j-1)} e^{H}}\right)
$$

and for $\cos (\bar{a})<0$, the limits are interchanged. The expression (6.10) corresponds exactly to one soliton solution, however expression (6.9) is multiplied by a complex constant $\Gamma_{1}$ and has $H$ shifted by $\ln \left(\Gamma_{N, c}\right)$. Thus, for $\cos (\bar{a})>0 x_{H}^{-}=0$ and $x_{H}^{+}=-\frac{\ln \left(\Gamma_{N, c}\right)}{2 \mu(-1)^{n} \sin \left(\frac{\pi d}{r}\right) \cosh (S)}$. Then

$$
\Delta_{H}(x)=\frac{-(-1)^{n} \operatorname{sign}(\cos (\bar{a})) \ln \left(\Gamma_{N, c}\right)}{2 \mu \cosh (S) \sin \left(\frac{\pi d}{r}\right)}
$$

where

$$
\Gamma_{N, c}=\frac{\cosh (S-\bar{b})-(-1)^{n} \sin \left(\bar{a}+\frac{\pi d}{r}\right)}{\cosh (S-\bar{b})-(-1)^{n} \sin \left(\bar{a}-\frac{\pi d}{r}\right)}
$$

For $(-1)^{n} \operatorname{sign}(\cos (\bar{a}))=+1$, we have $\Gamma_{N, c} \in\left[0,1\left[\right.\right.$ and hence $\ln \left(\Gamma_{N, c}\right)<0$. On the other hand, if $(-1)^{n} \operatorname{sign}(\cos (\bar{a}))=-1, \Gamma_{N, c} \in\left[1, \infty\left[\right.\right.$ and $\ln \left(\Gamma_{N, c}\right)>0$. Therefore, we can write both cases in a compact form

$$
\Delta_{H}(x)=\frac{\left|\ln \left(\Gamma_{N, c}\right)\right|}{2 \mu \cosh (S) \sin \left(\frac{\pi d}{r}\right)} \geq 0
$$

Following the arguments of ref. [17] and under the kinematics assumed above, $\Delta_{H}(x)>0$ implies that the force is attractive.

Under the same conditions $\tanh (S)>0, \tanh (S)-\tanh (\bar{b})>0$ but following the 1-soliton associated to $F$ :

$$
x-t \tanh (\bar{b})=\text { const }
$$

we obtain

$$
\Delta_{F}(x)=-\frac{\left|\ln \left(\Gamma_{N, c}\right)\right|}{2 \mu \cosh (\bar{b})|\cos (\bar{a})|}
$$

Reversing the conditions $\tanh (\bar{b})>0, \tanh (\bar{b})-\tanh (S)>0, \Delta_{H} \rightarrow-\Delta_{H}, \Delta_{F} \rightarrow-\Delta_{F}$. The time delay is for $\tanh (S)>0, \tanh (S)-\tanh (\bar{b})>0$

$$
\Delta_{H}(t)=-\frac{\left|\ln \left(\Gamma_{N, c}\right)\right|}{2 \mu \sinh (S) \sin \left(\frac{\pi d}{r}\right)}, \quad \Delta_{F}(t)=\frac{\left|\ln \left(\Gamma_{N, c}\right)\right|}{2 \mu \sinh (\bar{b})|\cos (\bar{a})|}
$$

Reversing the conditions for $(S, \bar{b})$ leads to $\Delta_{H}(t) \rightarrow-\Delta_{H}(t), \Delta_{F}(t) \rightarrow-\Delta_{F}(t)$. The same analysis can be done for the product $(\psi \chi)$. A subtle point is that if we follow the 1-soliton associated to $H$, we get $\lim _{t \rightarrow \pm \infty} \psi \chi=0$ which corresponds to the abelian 1-soliton and does not define the time delay. Following the 1-soliton associated to $F$, we get the corresponding $(\psi \chi)$ of the charged 1-soliton solution in (5.10) with $K=w^{d}$ and space displacement and time delay given by the same expressions above.

The analysis of the lateral displacements and time delays for the abelian neutral-neutral case is similar, replacing $\Gamma_{N, c}$ by $\Gamma_{N, N}$ interaction constant given by

$$
\Gamma_{N, N}=\frac{\cosh \left(S_{2}-S_{1}\right)-(-1)^{n_{2}-n_{1}} \cos \left(\frac{\pi\left(d_{1}-d_{2}\right)}{r}\right)}{\cosh \left(S_{2}-S_{1}\right)-(-1)^{n_{2}-n_{1}} \cos \left(\frac{\pi\left(d_{1}+d_{2}\right)}{r}\right)}
$$


where $d_{1}, d_{2}, S_{1}, S_{2}$ are defined in Appendix C. The associated time delay in the CMF is again negative leading to attractive forces.

Consider the solution given in (11.16) corresponding to the charged-charged case. Taking $v_{F_{1}}=\tanh (\hat{a})>0, \tanh (\hat{a})>\tanh (\hat{f})$ and following the 1-soliton associated to $F_{1}$ :

$$
x-t \tanh (\hat{a})=\text { const }
$$

we get

$$
\Delta_{F_{1}}(x)=-\frac{\operatorname{sign}(\cos (\bar{f})) \ln \left(\Gamma_{c, c}\right)}{2 \mu \cosh (\hat{a}) \cos (\bar{a})}
$$

where

$$
\Gamma_{c, c}=\frac{(\cosh (\hat{a}-\hat{f})-\cos (\bar{a}-\bar{f}))(\cosh (r(\hat{a}-\hat{f}))-\cos (r(\bar{a}-\bar{f})))}{(\cosh (\hat{a}-\hat{f})+\cos (\bar{a}+\bar{f}))\left(\cosh (r(\hat{a}-\hat{f}))-(-1)^{r} \cos (r(\bar{a}+\bar{f}))\right)}
$$

Analysing the last two eqns. we conclude that in general $\Delta_{F_{1}}(x)$ may be positive or negative. Therefore this solution represents 2 -soliton solution composed of atractive or repulsive forces acting between two charged 1-solitons.

\section{Bound States}

The scattering of two basic 1-solitons gives rise to the 2-soliton and bound state solutions. The most important diference between this last two kind of solutions is that the bound states have energy smaller than the corresponding 2-soliton solution. Their difference defines the binding energy.

As explained in the previous section we can obtain three classes of basic bound states: neutral-neutral (abelian), neutral-charged and charged-charged. We will discuss here the specific parametrization to the neutral-charged case. The final results are shown in the appendix $\mathrm{C}$ for all the cases. Consider the group element $g$ given by (6.1) and the corresponding tau functions (4.18). According to (12.9), (12.10) and (6.3), the energy and momentum are proportional to linear combinations of $\partial_{x} A_{i}\left(\gamma_{i}\right)$ and $\partial_{t} A_{i}\left(\gamma_{i}\right)$. The most simple choice to obtain real $(E, P)$ is to take $A_{1}\left(\gamma_{1}\right)+A_{2}\left(\gamma_{2}\right)$ and $A_{3}\left(\gamma_{3}\right)$ real giving rise to 2-soliton solutions. However there is another alternative by taking $A_{1}\left(\gamma_{1}\right)+A_{2}\left(\gamma_{2}\right)$ and $A_{3}\left(\gamma_{3}\right)$ complex but the combinations $A_{1}\left(\gamma_{1}\right)+A_{2}\left(\gamma_{2}\right) \pm A_{3}\left(\gamma_{3}\right)$ to be real. This leads to

$$
\begin{gathered}
A_{3} \rightarrow 2 \mu(-1)^{n} \sin \left(\frac{\pi d}{r}\right) \cosh (S)[(x-t \tanh (S)) \cos (q)+i(-t+x \tanh (S)) \sin (q)] \\
A_{1}+A_{2} \rightarrow F_{1}+F_{2}
\end{gathered}
$$

where

$$
\begin{gathered}
F_{1}=\mu \cosh (S)[(x-t \tanh (S)) \cos (\bar{a}+\tilde{a})+i(t-x \tanh (S)) \sin (\bar{a}+\tilde{a})], \\
F_{2}=F_{1}(\tilde{a} \rightarrow-\tilde{a} ; i \rightarrow-i)
\end{gathered}
$$


with the constraint

$$
\sin \left(\frac{\pi d}{r}\right) \sin (q)+\operatorname{Sign}\left[(-1)^{n} \cos (q) \cos (\bar{a}) \cos (\tilde{a})\right] \cos (\bar{a}) \sin (\tilde{a})=0
$$

The corresponding $(E, P, M)$ are given by

$$
M=\frac{E}{\cosh (S)}=\frac{P}{\sinh (S)}=\frac{2 k r \mu}{\pi}\left(|\cos (\bar{a}) \cos (\tilde{a})|+|\cos (q)| \sin \left(\frac{\pi d}{r}\right)\right)
$$

This defines the binding energy

$$
\Delta E=\frac{2 k r \mu}{\pi} \cosh (S)\left(|\cos (\bar{a})|(|\cos (\tilde{a})|-1)+\sin \left(\frac{\pi d}{r}\right)(|\cos (q)|-1)\right)
$$

The electric charge is also real and is given by

$$
Q_{e l}=-\frac{2 k r}{\pi}\left(\operatorname{sign}(\cos (\bar{a}) \cos (\tilde{a}))\left[\operatorname{sign}(\bar{a}) \frac{\pi}{2}-\bar{a}\right]-\left(n_{1}^{+}-n_{1}^{-}\right) \frac{\pi}{r}\right)
$$

for $\cos (\bar{a}) \cos (\tilde{a}) \neq 0$. The $(E, P, M)$ for the other two bound states are

$$
M=\frac{E}{\cosh (S)}=\frac{P}{\sinh (S)}=\frac{2 k r \mu}{\pi}\left(\sin \left(\frac{\pi d_{1}}{r}\right)\left|\cos \left(q_{1}\right)\right|+\sin \left(\frac{\pi d_{2}}{r}\right)\left|\cos \left(q_{2}\right)\right|\right)
$$

for the neutral-neutral solution. For the charged-charged case and under the conditions $\operatorname{sign}(\cos (\bar{a}+\tilde{a}))=\operatorname{sign}(\cos (\bar{a}-\tilde{a}))$ and $\operatorname{sign}(\cos (\bar{f}+\tilde{f}))=\operatorname{sign}(\cos (\bar{f}-\tilde{f}))$

$$
M=\frac{E}{\cosh (\hat{a})}=\frac{P}{\sinh (\hat{a})}=\frac{2 k r \mu}{\pi}(|\cos (\bar{a}) \cos (\tilde{a})|+|\cos (\bar{f}) \cos (\tilde{f})|)
$$

Other conditions can be analysed following the same procedure. The abelian case has zero electric charge while within the charged-charged case two different contributions appear according to the combinations

$$
\cos (\bar{a}) \cos (\tilde{a})>0(<0) \text { and } \cos (\bar{f}) \cos (\tilde{f})>0(<0) .
$$

\section{Outlook and Further Developments}

In this paper, the soliton solutions of the axial $A_{r}^{(1)}$ non abelian affine Toda model were constructed and classified in terms of vertex operators within the dressing formalism. Those axial models are characterized by the presence of an antisymmetric term (torsion term, see eqns. (2.17) and (2.18)). To each axial NA affine Toda model, a T-dual counterpart, torsionless model were obtained systematically in terms of vector gauging of the two-loop WZW model [25]. Their 1-soliton solution for the $A_{r}^{(1)}$ case is presented in [9].

A subclass of T-selfdual non abelian affine Toda models have been established in connection with Kac-Moody algebras whose Dynkin diagram possess a " $B_{r}$-tail like" [25], namely $B_{r}^{(1)}, A_{2 r}^{(2)}$ and $D_{r+1}^{(2)}$ and coincide precisely with the models discussed by Fateev [26].

A general study and classification of the soliton solutions of the torsionless T-dual and T-selfdual models in terms of dressing formalism, $\tau$-functions and vertex operators are still to be completed and shall be reported elsewhere.

Acknowledgements We thank L.A. Ferreira for many useful discussions. The financial support of CNPq, Fapesp and Unesp is gratefully acknowleged. 


\section{Appendix A}

Here we give an example of a typical calculation required to determine the tau functions. Consider $g=\exp \left[a F_{1, r}^{-}\left(\gamma_{1}\right)\right] \exp \left[b F_{1, r}^{+}\left(\gamma_{2}\right)\right]$. Then since $T_{0}=\exp \left(-z \epsilon_{-}\right) \exp \left(\bar{z} \epsilon_{+}\right)$and

$$
\left[\epsilon^{ \pm}, F_{1, r}^{+}\left(\gamma_{2}\right)\right]=-\mu \gamma_{2}^{ \pm} F_{1, r}^{+}\left(\gamma_{2}\right), \quad\left[\epsilon^{ \pm}, F_{1, r}^{-}\left(\gamma_{1}\right)\right]=\mu \gamma_{1}^{ \pm} F_{1, r}^{-}\left(\gamma_{1}\right)
$$

we get

$$
T_{0} g T_{0}^{-1}=\exp \left[a A_{1}\left(\gamma_{1}\right) F_{1, r}^{-}\left(\gamma_{1}\right)\right] \exp \left[b A_{2}\left(\gamma_{2}\right) F_{1, r}^{+}\left(\gamma_{2}\right)\right]
$$

where

$$
A_{1}\left(\gamma_{1}\right)=\left(-\frac{\mu z}{\gamma_{1}}+\mu \bar{z} \gamma_{1}\right), \quad A_{2}\left(\gamma_{2}\right)=A_{1}\left(\gamma_{1} \rightarrow-\gamma_{2}\right)
$$

Let us now calculate

$$
\begin{aligned}
\tau_{0} & =\left\langle\lambda_{0}\left|T_{0} g T_{0}^{-1}\right| \lambda_{0}\right\rangle \\
& =\left\langle\lambda_{0}\left|1+a A_{1}\left(\gamma_{1}\right) F_{1, r}^{-}+b A_{2}\left(\gamma_{2}\right) F_{1, r}^{+}\left(\gamma_{2}\right)+a b A_{1}\left(\gamma_{1}\right) A_{2}\left(\gamma_{2}\right) F_{1, r}^{-}\left(\gamma_{1}\right) F_{1, r}^{+}\left(\gamma_{2}\right)\right| \lambda_{0}\right\rangle
\end{aligned}
$$

The last step cames from the fact that $F_{1, r}^{ \pm}$are associated to (nilpotent) vertex operators, i.e., their square inside the matrix element vanish. Let

$$
F_{1, r}^{ \pm}(\gamma)=\sum_{n=-\infty}^{\infty} \gamma^{-n r} \sum_{p=0}^{r-1} \gamma^{\mp p} E_{ \pm\left(\alpha_{1}+\cdots+\alpha_{p+1}\right)}^{(n)}
$$

We see that

$$
\left\langle\lambda_{0}|I| \lambda_{0}\right\rangle=1, \quad\left\langle\lambda_{0}\left|F_{1, r}^{ \pm}(\gamma)\right| \lambda_{0}\right\rangle=0
$$

where we have used the fact that $E_{\left(\alpha_{1}+\cdots+\alpha_{p+1}\right)}^{(n)}\left|\lambda_{0}\right\rangle=0, n \geq 0$. Also $\left\langle\lambda_{0}\right| E_{-\left(\alpha_{1}+\cdots+\alpha_{p+1}\right)}^{(n)}=$ $0, n \leq 0$ and $\left[E_{-\left(\alpha_{1}+\cdots+\alpha_{p+1}\right)}^{(n)}, E_{\left(\alpha_{1}+\cdots+\alpha_{p+1}\right)}^{(n)}\right]=n \hat{c} \delta_{m+n, 0}-\sum_{j=1}^{p+1} h_{j}^{(m+n)}$, leading to

$$
\left\langle\lambda_{0}\left|F_{1, r}^{-}\left(\gamma_{1}\right) F_{1, r}^{+}\left(\gamma_{2}\right)\right| \lambda_{0}\right\rangle=\sum_{n>0} n \gamma_{2,1}^{r n} \sum_{p=0}^{r-1} \gamma_{1,2}^{p}=\frac{\gamma_{2,1}^{r}}{\left(1-\gamma_{2,1}^{r}\right)^{2}} \frac{\left(1-\gamma_{1,2}^{r}\right)}{\left(1-\gamma_{1,2}\right)}
$$

where $\gamma_{i j}=\frac{\gamma_{i}}{\gamma_{j}}$.

\section{Appendix B}

In this appendix we want to show that the obtained tau functions do not depend upon the order in which the vertices are put in the group element $g$. To be more precise, let $g=\Pi_{i=1}^{N} e^{\alpha_{i} F_{i}\left(\gamma_{i}\right)}$, with $N$ arbitrary and $F_{i} \in\left\{F_{1, r}^{ \pm}, F_{d, r}\right\}$ and define

$$
\begin{aligned}
\sigma_{j, k} g & =\sigma_{j, k}\left\{e^{\alpha_{1} F_{1}\left(\gamma_{1}\right)} \cdots e^{\alpha_{j} F_{j}\left(\gamma_{j}\right)} \cdots e^{\alpha_{k} F_{k}\left(\gamma_{k}\right)} \cdots e^{\alpha_{N} F_{N}\left(\gamma_{N}\right)}\right\} \\
& =\left\{e^{\alpha_{1} F_{1}\left(\gamma_{1}\right)} \cdots e^{\alpha_{k} F_{k}\left(\gamma_{k}\right)} \cdots e^{\alpha_{j} F_{j}\left(\gamma_{j}\right)} \cdots e^{\alpha_{N} F_{N}\left(\gamma_{N}\right)}\right\}
\end{aligned}
$$

for $j \neq k, j, k=1, \cdots, N$. We want to show that $\tau(g)=\tau\left(\sigma_{j k}(g)\right)$, where $\tau=\left(\tau_{0}, \tau_{R}, \tau_{j}, \tau_{\psi}, \tau_{\chi}\right)$. 
Let us note that the vertex $F_{i}$ can be written as $F_{i}=\sum_{n=-\infty}^{\infty} O(n) z^{n}$, where $z$ is some complex constant and $O(n)$ are linear combinations of the generators with the $n$ dependence comming only from $\left(E_{\alpha}^{(n)}, h_{i}^{(n)}\right)$. Consider $\tau \neq\left(\tau_{\psi}, \tau_{\chi}\right)$, then

$$
\tau(g)-\tau\left(\sigma_{j, j+1}(g)\right)=\left\langle\lambda_{l}\left|\Pi_{i=1}^{N} e^{a_{i} A_{i}\left(\gamma_{i}\right) F_{i}\left(\gamma_{i}\right)}\right| \lambda_{l}\right\rangle-\left\langle\lambda_{l}\left|\sigma_{j, j+1} \Pi_{i=1}^{N} e^{a_{i} A_{i}\left(\gamma_{i}\right) F_{i}\left(\gamma_{i}\right)}\right| \lambda_{l}\right\rangle
$$

where $l=0, \cdots, r$ and $A_{i}\left(\gamma_{i}\right)$ are certain functions of $\gamma_{i}$. Then we can expand the exponentials and to join the corresponding terms. For example, the last terms,

$$
\begin{aligned}
& \left\langle\lambda_{l}\left|\Pi_{i=1}^{N} a_{i} A_{i}\left(\gamma_{i}\right) F_{i}\left(\gamma_{i}\right)\right| \lambda_{l}\right\rangle-\left\langle\lambda_{l}\left|\sigma_{j, j+1} \Pi_{i=1}^{N} a_{i} A_{i}\left(\gamma_{i}\right) F_{i}\left(\gamma_{i}\right)\right| \lambda_{l}\right\rangle \\
= & a_{1}^{2} \cdots a_{n}^{2} A_{1}^{2}\left(\gamma_{1}\right) \cdots A_{N}^{2}\left(\gamma_{N}\right)\left\langle\lambda_{l}\left|F_{1}\left(\gamma_{1}\right) \cdots\left[F_{j}\left(\gamma_{j}\right), F_{j+1}\left(\gamma_{j+1}\right)\right] \cdots F_{N}\left(\gamma_{N}\right)\right| \lambda_{l}\right\rangle
\end{aligned}
$$

This expression corresponds to the sum of products of $N-1$ operators, since the commutator is equivalent to one operator. But, there are $N$ integer dummy variables (summed from $-\infty$ to $\infty$ ), one for each vertex. The standard algorithm to calculate the Kac-Moody matrix element is to reduce step by step the product of $N-1$ operators to one single operator that can be evaluated. At each step of this process one reduces the number of unrestricted independent dummy variables (i.e. $]-\infty, \infty[$ ) by one. At the end of the process we have $N-1$ restricted (range $\neq]-\infty, \infty[$ ) dummy variables and one unrestricted. From the KacMoody commutation relations one can see that there are only terms proportional to $z^{\prime n}$ or $n z^{\prime n}$ for $n$ unrestricted ( $z^{\prime}$ some complex constant) and therefore according to the relation

$\sum_{n=-\infty}^{\infty} z^{\prime n}=\sum_{n=-\infty}^{\infty} n z^{\prime n}=0$ leads to the vanishing of (10.3). The same argument can be used to show the vanishing of (10.2). Also it is possible to do the same to $\left(\tau_{\psi}, \tau_{\chi}\right)$.

\section{Appendix C}

In this appendix we give the explicit solution of the three kinds of 2 -solitons and bound states.

a)neutral-neutral: This solution is obtained from $g=\exp \left[a F_{d_{1}, r}\left(\gamma_{1}\right)\right] \exp \left[b F_{d_{2}, r}\left(\gamma_{2}\right)\right]$

$$
\begin{aligned}
\tau_{0} & =1+e^{\zeta_{1}+H_{1}}+e^{\zeta_{2}+H_{2}}+\Gamma_{N, N} e^{\zeta_{1}+\zeta_{2}} e^{H_{1}+H_{2}} \\
\tau_{j} & =1+w^{d_{1}(j-1)} e^{\zeta_{1}+H_{1}}+w^{d_{2}(j-1)} e^{\zeta_{2}+H_{2}}+\Gamma_{N, N} w^{\left(d_{1}+d_{2}\right)(j-1)} e^{\zeta_{1}+\zeta_{2}} e^{H_{1}+H_{2}}, j=2, \cdots r \\
\tau_{R} & =\tau_{0}, \quad \tau_{\psi}=\tau_{\chi}=0
\end{aligned}
$$

where $d_{1}, d_{2}=1, \cdots r-1, \zeta_{1}, \zeta_{2} \in C, w=e^{\frac{2 \pi i}{r}}$. For the 2-soliton

$$
\begin{aligned}
H_{k} & =2 \mu(-1)^{n_{k}} \sin \left(\frac{\pi d_{k}}{r}\right) \cosh \left(S_{k}\right)\left(x-t \tanh \left(S_{k}\right)\right), \quad k=1,2 \\
\Gamma_{N, N} & =\frac{\cosh \left(S_{1}-S_{2}\right)-(-1)^{\left(n_{2}-n_{1}\right)} \cos \left(\frac{\pi}{r}\left(d_{1}-d_{2}\right)\right)}{\cosh \left(S_{1}-S_{2}\right)-(-1)^{\left(n_{2}-n_{1}\right)} \cos \left(\frac{\pi}{r}\left(d_{1}+d_{2}\right)\right)}, \quad S_{k} \in R, \quad n_{k} \in Z
\end{aligned}
$$

For the bound state,

$$
\begin{aligned}
H_{k} & =2 \mu(-1)^{n_{k}} \sin \left(\frac{\pi d_{k}}{r}\right) \cosh (S)\left((x-t \tanh (S)) \cos \left(q_{k}\right)\right. \\
& \left.+i(-t+x \tanh (S)) \sin \left(q_{k}\right)\right), \\
\Gamma_{N, N}^{(b)} & =\Gamma_{N, N}^{(2-s)}\left(S_{2}-S_{1} \rightarrow i\left(q_{2}-q_{1}\right)\right), \quad S, q_{2}, q_{1} \in R
\end{aligned}
$$


with the constraint

$$
\sin \left(\frac{\pi d_{1}}{r}\right) \sin \left(q_{1}\right)+\operatorname{Sign}\left[(-1)^{\left(n_{1}+n_{2}\right)} \cos \left(q_{1}\right) \cos \left(q_{2}\right)\right] \sin \left(\frac{\pi d_{2}}{r}\right) \sin \left(q_{2}\right)=0
$$

b) Neutral-Charged:This solution is obtained from

$$
\begin{aligned}
g=\exp \left[a F_{1, r}^{-}\left(\gamma_{1}\right)\right] \exp \left[b F_{1, r}^{+}\left(\gamma_{2}\right)\right] \exp \left[c F_{d, r}\left(\gamma_{3}\right)\right] \\
\tau_{0}=1+e^{\zeta_{1}+H}+e^{F_{1}+F_{2}} e^{\zeta_{2}}+\Gamma_{N, c} e^{\zeta_{1}+\zeta_{2}} e^{H+F_{1}+F_{2}} \\
\tau_{R}=1+e^{\zeta_{1}+H}+\Gamma_{1}^{-r} e^{F_{1}+F_{2}} e^{\zeta_{2}}+\Gamma_{1}^{-r} \Gamma_{N, c} e^{\zeta_{1}+\zeta_{2}} e^{H+F_{1}+F_{2}} \\
\tau_{j}=1+w^{d(j-1)} e^{\zeta_{1}+H}+\Gamma_{1}^{-r+j-1} e^{F_{1}+F_{2}} e^{\zeta_{2}}+w^{d(j-1)} \Gamma_{1}^{-r+j-1} \Gamma_{N, c} e^{\zeta_{1}+\zeta_{2}} e^{H+F_{1}+F_{2}} \\
\tau_{\chi}==\Gamma_{2} e^{\theta+\frac{1}{2} \zeta_{2}} e^{F_{1}}\left(1+\Gamma_{3} e^{\zeta_{1}+H}\right) \\
\tau_{\psi}=\Gamma_{2} e^{-\theta+\frac{1}{2} \zeta_{2}} e^{F_{2}}\left(1+\Gamma_{4} e^{\zeta_{1}+H}\right)
\end{aligned}
$$

where $d=1, \cdots, r-1, \theta, \zeta_{1}, \zeta_{2} \in C, \Gamma_{1}, \Gamma_{2}$ are defined in (5.11).

For the 2-soliton.

$$
\begin{gathered}
F_{1}=F+i G, F_{2}=F-i G, \\
F=\mu \cos (\bar{a}) \cosh (\bar{b})(x-t \tanh (\bar{b})) \\
G=\mu \sin (\bar{a}) \cosh (\bar{b})(t-x \tanh (\bar{b})) \\
H=2 \mu(-1)^{n} \sin \left(\frac{\pi d}{r}\right) \cosh (S)(x-t \tanh (S)) \\
\Gamma_{3}^{(2-s)}=w^{d} \frac{1-i(-1)^{n} e^{-(S-\bar{b})} e^{-i \bar{a}} w^{-\frac{1}{2} d}}{1-i(-1)^{n} e^{-(S-\bar{b})} e^{-i \bar{a}} w^{\frac{1}{2} d}}, \quad \Gamma_{4}^{(2-s)}=\Gamma_{3}^{(2-s)}((S-\bar{b}) \rightarrow-(S-\bar{b})) \\
\Gamma_{N, c}^{(2-s)} \equiv w^{-d} \Gamma_{3}^{(2-s)} \Gamma_{4}^{(2-s)}=\frac{\cosh (S-\bar{b})-(-1)^{n} \sin \left(\bar{a}+\frac{\pi d}{r}\right)}{\cosh (S-\bar{b})-(-1)^{n} \sin \left(\bar{a}-\frac{\pi d}{r}\right)}, \quad S, \bar{b}, \bar{a} \in R, n \in Z
\end{gathered}
$$

For the bound state

$$
\begin{gathered}
\Gamma_{3}^{(b)}=\Gamma_{3}^{(2-s)}(S-\bar{b} \rightarrow i(\tilde{a}+q)), \quad \Gamma_{4}^{(b)}=\Gamma_{3}^{(b)}(i(\tilde{a}+q) \rightarrow-i(\tilde{a}+q)) \\
\Gamma_{N, c}^{(b)}=\Gamma_{N, c}^{(2-s)}(S-\bar{b} \rightarrow i(\tilde{a}+q)), \quad \tilde{a}, q \in R \\
F_{1}=\mu \cosh (S)[(x-t \tanh (S)) \cos (\bar{a}+\tilde{a})+i(t-x \tanh (S)) \sin (\bar{a}+\tilde{a})] \\
H=2 \mu(-1)^{n} \sin \left(\frac{\pi d}{r}\right) \cosh (S)[(x-t \tanh (S)) \cos (q)+i(-t+x \tanh (S)) \sin (q)]
\end{gathered}
$$

$F_{2}=F_{1}(\tilde{a} \rightarrow-\tilde{a} ; i \rightarrow-i)$ with the constraint

$$
\sin \left(\frac{\pi d}{r}\right) \sin (q)+\operatorname{sign}\left[(-1)^{n} \cos (q) \cos (\bar{a}) \cos (\tilde{a})\right] \cos (\bar{a}) \sin (\tilde{a})=0
$$


c) Charged-Charged:This solution is obtained from

$$
\begin{aligned}
& g=\exp \left[a F_{1, r}^{-}\left(\gamma_{a}\right)\right] \exp \left[b F_{1, r}^{+}\left(\gamma_{b}\right)\right] \exp \left[c F_{1, r}^{-}\left(\gamma_{f}\right)\right] \exp \left[g F_{1, r}^{+}\left(\gamma_{g}\right)\right] \\
\tau_{0}= & 1+e^{\zeta_{1}+F_{1}+F_{3}}+e^{F_{2}+F_{4}} e^{\zeta_{2}}+K_{1} e^{F_{1}+F_{4}}+K_{2} e^{F_{2}+F_{3}}+\Gamma_{c, c} e^{F_{1}+F_{2}+F_{3}+F_{4}} e^{\zeta_{1}+\zeta_{2}} \\
\tau_{R}= & 1+\Gamma_{1}^{-r} e^{F_{1}+F_{3}} e^{\zeta_{1}}+\Gamma_{2}^{-r} e^{\zeta_{2}} e^{F_{2}+F_{4}}+\Gamma_{3}^{-r} K_{1} e^{F_{1}+F_{4}} \\
+ & \Gamma_{4}^{-r} K_{2} e^{F_{2}+F_{3}}+\left(\Gamma_{1} \Gamma_{2}\right)^{-r} \Gamma_{c, c} e^{F_{1}+F_{2}+F_{3}+F_{4}} e^{\zeta_{1}+\zeta_{2}} \\
\tau_{j}= & \tau_{R}\left(\Gamma_{i}^{-r} \rightarrow \Gamma_{i}^{j-r-1}\right), i=2, \cdots r \\
\tau_{\chi}= & e^{\theta_{1}+\frac{1}{2} \zeta_{1}} \Omega_{1} e^{F_{1}}+e^{\theta_{2}+\frac{1}{2} \zeta_{2}} \Omega_{2} e^{F_{2}}+A_{1} e^{F_{1}+F_{2}+F_{4}}+A_{2} e^{F_{1}+F_{2}+F_{3}} \\
\tau_{\psi}= & e^{-\theta_{1}+\frac{1}{2} \zeta_{1}} \Omega_{1} e^{F_{3}}+e^{-\theta_{2}+\frac{1}{2} \zeta_{2}} \Omega_{2} e^{F_{4}}+A_{3} e^{F_{1}+F_{3}+F_{4}}+A_{4} e^{F_{2}+F_{3}+F_{4}}
\end{aligned}
$$

where

$$
\begin{aligned}
\Gamma_{1} & =\gamma_{a, b}, \quad \Gamma_{2}=\gamma_{f, g} \quad \Gamma_{3}=\gamma_{a, g} \quad \Gamma_{4}=\gamma_{f, b} \quad \Gamma_{5}=\gamma_{b, g} \\
\Omega_{i} & =\sqrt{\left(1-\Gamma_{i}\right)\left(\Gamma_{i}^{-r}-1\right)}, \quad i=1,2, \quad \Omega_{j}^{-1}=\sqrt{\left(1-\Gamma_{j}\right)\left(\Gamma_{j}^{-r}-1\right)}, \quad j=3,4, \\
K_{1} & =e^{\theta_{1}-\theta_{2}} e^{\frac{1}{2}\left(\zeta_{1}+\zeta_{2}\right)} \Omega_{1} \Omega_{2} \Omega_{3}^{2}, \quad K_{2}=e^{\theta_{2}-\theta_{1}} e^{\frac{1}{2}\left(\zeta_{1}+\zeta_{2}\right)} \Omega_{1} \Omega_{2} \Omega_{4}^{2}
\end{aligned}
$$

where $\zeta_{1}, \zeta_{2}, \theta_{1}, \theta_{2} \in C$, and

$$
\begin{aligned}
& A_{1}=e^{\theta_{1}+\frac{1}{2} \zeta_{1}+\zeta_{2}}\left(\Gamma_{2}-\Gamma_{3}\right)\left(\Gamma_{2}^{r}-\Gamma_{3}^{r}\right)\left(\Gamma_{2} \Gamma_{3}\right)^{-r} \Omega_{1} \Omega_{3}^{2} \\
& A_{2}=e^{\theta_{2}+\frac{1}{2} \zeta_{2}+\zeta_{1}}\left(\Gamma_{4}-\Gamma_{1}\right)\left(\Gamma_{4}^{r}-\Gamma_{1}^{r}\right)\left(\Gamma_{1} \Gamma_{4}\right)^{-r} \Omega_{2} \Omega_{4}^{2} \\
& A_{3}=e^{-\theta_{2}+\frac{1}{2} \zeta_{2}+\zeta_{1}}\left(\Gamma_{3}-\Gamma_{1}\right)\left(\Gamma_{3}^{r}-\Gamma_{1}^{r}\right)\left(\Gamma_{1} \Gamma_{3}\right)^{-r} \Omega_{2} \Omega_{3}^{2} \\
& A_{4}=e^{-\theta_{1}+\frac{1}{2} \zeta_{1}+\zeta_{2}}\left(\Gamma_{2}-\Gamma_{4}\right)\left(\Gamma_{2}^{r}-\Gamma_{4}^{r}\right)\left(\Gamma_{2} \Gamma_{4}\right)^{-r} \Omega_{1} \Omega_{4}^{2}
\end{aligned}
$$

For the 2-soliton, $F_{1}=F+i G, \quad F_{3}=F-i G$

$$
\begin{gathered}
F=\mu \cos (\bar{a}) \cosh (\hat{a})(x-t \tanh (\hat{a})), \\
G=\mu \sin (\bar{a}) \cosh (\hat{a})(t-x \tanh (\hat{a})), \\
\left(F_{2}, F_{4}\right)=\left(F_{1}, F_{3}\right)(\bar{a} \rightarrow \bar{f}, \hat{a} \rightarrow \hat{f}) \\
\gamma_{a}=-e^{\hat{a}-i \bar{a}}, \quad \gamma_{b}=e^{\hat{a}+i \bar{a}}, \quad \gamma_{f}=-e^{\hat{f}-i \bar{f}}, \quad \gamma_{g}=e^{\hat{f}+i \bar{f}} \\
\Gamma_{c, c}^{(2-s)}=\frac{(\cosh (\hat{a}-\hat{f})-\cos (\bar{a}-\bar{f}))(\cosh (r(\hat{a}-\hat{f}))-\cos (r(\bar{a}-\bar{f})))}{(\cosh (\hat{a}-\hat{f})+\cos (\bar{a}+\bar{f}))\left(\cosh (r(\hat{a}-\hat{f}))-(-1)^{r} \cos (r(\bar{a}+\bar{f}))\right)}
\end{gathered}
$$

where $\bar{a}, \hat{a}, \bar{f}, \hat{f} \in R$.

For the bound state

$$
\begin{aligned}
F_{1} & =\mu \cosh (\hat{a})((x-t \tanh (\hat{a})) \cos (\bar{a}+\tilde{a})+i(t-x \tanh (\hat{a})) \sin (\bar{a}+\tilde{a})) \\
F_{3} & =F_{1}(\tilde{a} \rightarrow-\tilde{a} ; i \rightarrow-i), \quad F_{2}=F_{1}(\tilde{a} \rightarrow \tilde{f}, \bar{a} \rightarrow \bar{f}), \quad F_{4}=F_{1}(\tilde{a} \rightarrow-\tilde{f}, \bar{a} \rightarrow \bar{f} ; i \rightarrow-i) \\
\gamma_{a} & =-e^{\hat{a}-i(\bar{a}+\tilde{a})}, \quad \gamma_{b}=e^{\hat{a}+i(\bar{a}-\tilde{a})}, \quad \gamma_{f}=-e^{\hat{a}-i(\bar{f}+\tilde{f})}, \quad \gamma_{g}=e^{\hat{a}+i(\bar{f}-\tilde{f})} \\
\Gamma_{c, c}^{(b)} & =\Gamma_{c, c}^{(2-s)}(\hat{a}-\hat{f} \rightarrow i(\tilde{a}-\tilde{f})), \quad \hat{a}, \bar{a}, \tilde{a}, \bar{f}, \tilde{f} \in R,
\end{aligned}
$$

with the constraint

$$
\cos (\bar{a}) \sin (\tilde{a})+\operatorname{sign}[\cos (\bar{a}) \cos (\tilde{a}) \cos (\bar{f}) \cos (\tilde{f})] \cos (\bar{f}) \sin (\tilde{f})=0
$$




\section{Appendix D}

In order to evaluate the energy and momentum of the system it is convenient to use the procedure of ref. [21]. The canonical energy-momentum tensor defined from the action (3.10) does not satisfy the traceless condition. Instead,

$$
T_{\alpha}^{\alpha}=2 V
$$

where $V$ is the potential appearing in the lagrangean density (3.10). Using the equations of motion (3.6)-(3.9), we find,

$$
T_{\alpha}^{\alpha}=4 \partial_{\rho} \partial^{\rho} f=4 \partial \bar{\partial} f
$$

where

$$
f=-\frac{k}{4 \pi}\left\{\sum_{i=2}^{r} \varphi_{i}+\frac{r(r-1)}{2(r+1)} R+r \nu\right\} .
$$

and therefore define a traceless conformal energy-momentun tensor

$$
\Theta_{c a t}^{\mu \nu}=T^{\mu \nu}+4\left(\partial^{\mu} \partial^{\nu}-g^{\mu \nu} \partial_{\rho} \partial^{\rho}\right) f
$$

In ref. [21] it is argued that the total energy and total momentum are given by

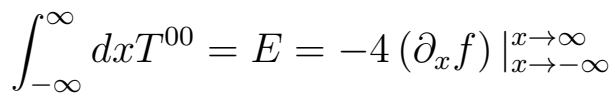

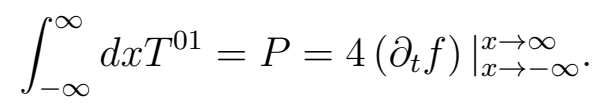

It follows that $e^{-\frac{4 \pi}{k} f}=\tau_{0} \tau_{2} \cdots \tau_{r} e^{r \mu^{2} z \bar{z}}$ and therefore for the energy momentum we have

$$
E=-\left(-\frac{k}{\pi}\right)\left[\frac{\partial_{x} \tau_{0}}{\tau_{0}}+\frac{\partial_{x} \tau_{2}}{\tau_{2}}+\ldots+\frac{\partial_{x} \tau_{r}}{\tau_{r}}+\frac{\partial_{x}\left(e^{r \mu^{2} z \bar{z}}\right)}{e^{r \mu^{2} z \bar{z}}}\right]_{x \rightarrow-\infty}^{x \rightarrow \infty}
$$

and

$$
P=\left(-\frac{k}{\pi}\right)\left[\frac{\partial_{t} \tau_{0}}{\tau_{0}}+\frac{\partial_{t} \tau_{2}}{\tau_{2}}+\ldots+\frac{\partial_{t} \tau_{r}}{\tau_{r}}+\frac{\partial_{t}\left(e^{r \mu^{2} z \bar{z}}\right)}{e^{r \mu^{2} z \bar{z}}}\right]_{x \rightarrow-\infty}^{x \rightarrow \infty}
$$

the last term in eqn (12.8) does not contributes, while in (12.7) contributes with an infinite value (zero point energy), which will be regularized.

From the explicit form of the solution, it is easy to check directly that each term in (12.7) and in (12.8) yields the same asymptotic contribution and hence

$$
\begin{gathered}
E=\frac{k r}{\pi}\left(\frac{\partial_{x} \tau_{0}}{\tau_{0}}\right)_{x \rightarrow-\infty}^{x \rightarrow \infty} \\
P=-\frac{k r}{\pi}\left(\frac{\partial_{t} \tau_{0}}{\tau_{0}}\right)_{x \rightarrow-\infty}^{x \rightarrow \infty} .
\end{gathered}
$$




\section{References}

[1] N. Ganoulis, P. Goddard, D. Olive Nucl. Phys. B205,(1982), 601

[2] A. Kovner, M. Shiffman and A. Smilga, Phys. Rev. D56, (1997),7978-7989, also hepth/9706089; G. Dvali and M. Shiffman, Phys. Lett. B396,(1997), hep-th/9612128

[3] A. Hanany and K. Hori, Nucl. Phys. B513 (1998) 119

[4] E. Witten, Nucl. Phys. B507 (1997) 658

[5] E. Witten, Nucl. Phys. B160 (1979) 461

[6] J. Gauntlett, D. Tong, P. Townsend, "Supersymmetric intersecting domain walls in massive hyperkahler sigma models", DAMTP-2000-69, hep-th/0007124; J. Gauntlett, R. Portugues, D. Tong, P. Townsend, "D-brane solitons in supersymmetric models", DAMTP-2000-68, hep-th/0008221;

[7] F. Lund, Ann. of Phys. 415 (1978) 251;

F. Lund and T. Regge, Phys. Rev. D14 (1976) 1524

[8] C.R. Fernandez-Pousa, M.V. Gallas, T.J. Hollowood and J.L. Miramontes, Nucl. Phys. B484 (1997) 609; Nucl. Phys. B499 (1997) 673; C.R. Fernandez-Pousa and J.L. Miramontes, Nucl. Phys. B518 (1998) 745; O. Castro Alvaredo, J.L. Miramontes Nucl. Phys. B581 (2000), 643-678, hep-th/0002219

[9] J.F. Gomes, E.P. Gueuvoghlanian, G.M. Sotkov and A.H. Zimerman, "Electrically Charged Topological Solitons", IFT preprint IFT-P.064/2000, hepth/0007169,

[10] A. N. Leznov, M. V. Saveliev, Group Theoretical Methods for Integration of Nonlinear Dynamical Systems, Progress in Physics, Vol. 15 (1992), Birkhauser Verlag, Berlin; A. N. Leznov and M. V. Saveliev, Commun. Math. Phys. 89 (1983) 59

[11] H. Aratyn, L.A. Ferreira, J.F. Gomes and A.H. Zimerman, Phys. Lett B 254 (1991) 372

[12] U. Harder, A. Iskandar, W. A. McGhee Int. J. Mod. Phys. A10,(1995),1879-1904 hepth/9409035

[13] L.A. Ferreira, J.L. Miramontes and J.S. Guillen, J. Math. Phys. 38, (1997),882-901; L.A. Ferreira, J.L. Miramontes and J.S. Guillen, Nucl. Phys. B449 (1995) 631-679;

[14] J.F. Gomes, E.P. Gueuvoghlanian, G.M. Sotkov and A.H. Zimerman, "Classical Integrability of Non Abelian Affine Toda Models, to appear in the Proc. of the XXIII International Colloquium on Group Theoretical Methods in Physics, Ed. G. Pogosyan et. al., Dubna (2000), hepth/0010257 
[15] J.F. Gomes, F.E.M. da Silveira, G.M. Sotkov and A.H. Zimerman, "Singular NonAbelian Toda Theories", hepth 9810057, in "Nonassociative Algebras and its Applications", Lec. Notes in Mathematics, Vol 211,p. 125-136 Ed. R. Costa et. al., Marcel Dekker, (1999); J.F. Gomes, E.P. Gueuvoghlanian, F.E.M. da Silveira, G.M. Sotkov and A.H. Zimerman, "Singular Conformal, and Conformal Affine Non-Abelian Toda Theories", "M.V. Saveliev Memorial Volume", Dubna (2000), Ed. A.N. Sissakian, p. 38-50, see also hepth/0002173.

[16] J.F. Gomes, G.M. Sotkov and A.H. Zimerman, Phys. Lett. 435B (1998) 49, hepth/9803122; J.F. Gomes, G.M. Sotkov and A.H. Zimerman, Ann. of Phys.274,(1999),289-362, hepth/9803234

[17] A. Fring, P.R. Johnson, M.A.C. Kneipp, D.I. Olive, Nucl.Phys. B430,(1994),597-614, hep-th/9405034.

[18] E. Witten, Phys. Rev. Lett. 38 (1978) 121

[19] J.-L. Gervais and M. V. Saveliev, Phys. Lett. 286B (1992) 271

[20] T. Hollowood, Nucl. Phys. B384 (1992) 523; H.C. Liao, D. Olive and N. Turok, Phys. Lett. 298B (1993) 95;

[21] H. Aratyn, C.P. Constantinides, L.A. Ferreira, J.F. Gomes and A.H. Zimerman, Nucl. Phys. B406(1993),727

[22] H. Aratyn, L.A. Ferreira, J.F. Gomes and A.H. Zimerman, J. Phys. A31, (1998) 94839492

[23] H. Aratyn, L.A. Ferreira, J.F. Gomes and A.H. Zimerman, Supersymmetry and Integrable models (Lecture Notes in Physics Vol. 502), Ed. H. Aratyn et. al. (Berlin, Springer), p. 197-210, (1998)

[24] N. Dorey and T.J. Hollowood, hep-th/9410140, Nucl.Phys. B440 (1995) 215-236

[25] J.F. Gomes, E.P. Gueuvoghlanian, G.M. Sotkov and A.H. Zimerman, "Torssionless T Selfdual Affine Non Abelian Toda Models", hep-th/0002173, To appear in the Proc. of the VI International Wigner Symposium, Istambul, Turkey, 2000; see also J.F. Gomes, E.P. Gueuvoghlanian, G.M. Sotkov and A.H. Zimerman, "T-duality of axial and vector dyonic integrable models", hep-th/0007116, to appear in Ann. of Phys. (2001)

[26] V. Fateev, Nucl. Phys. B479 (1996) 594 